\title{
Potential Roles for Ubiquitin and the Proteasome during Ribosome Biogenesisł
}

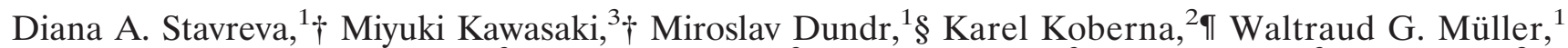 \\ Teruko Tsujimura-Takahashi, ${ }^{3}$ Wataru Komatsu, ${ }^{3}$ Toshiya Hayano, ${ }^{3}$ Toshiaki Isobe, ${ }^{3}$ Ivan Raska, ${ }^{2}$ \\ Tom Misteli, ${ }^{1}$ Nobuhiro Takahashi, ${ }^{3}$ and James G. McNally ${ }^{1 *}$ \\ Laboratory of Receptor Biology and Gene Expression, Center for Cancer Research, National Cancer Institute, Bethesda, Maryland 20892 ${ }^{1}$, \\ Institute of Cellular Biology and Pathology, First Faculty of Medicine, Charles University in Prague, and Institute of \\ Physiology, Academy of Sciences of the Czech Republic, Albertov 4, 12800 Prague 2, Czech Republic ${ }^{2}$; and \\ Department of Applied Biological Science and Department of Biotechnology, United Graduate School of \\ Agriculture, Tokyo University of Agriculture and Technology, and Integrated Proteomics System Project, \\ Pioneer Research on Genome the Frontier, Ministry of Education, Culture, Sports, Science, \\ and Technology of Japan, 3-5-8 Saiwai-cho, Fuchu-shi, Tokyo 183-8509, Japan ${ }^{3}$
}

Received 18 November 2005/Returned for modification 23 December 2005/Accepted 17 March 2006

\begin{abstract}
We have investigated the possible involvement of the ubiquitin-proteasome system (UPS) in ribosome biogenesis. We find by immunofluorescence that ubiquitin is present within nucleoli and also demonstrate by immunoprecipitation that complexes associated with pre-rRNA processing factors are ubiquitinated. Using short proteasome inhibition treatments, we show by fluorescence microscopy that nucleolar morphology is disrupted for some but not all factors involved in ribosome biogenesis. Interference with proteasome degradation also induces the accumulation of $90 \mathrm{~S}$ preribosomes, alters the dynamic properties of a number of processing factors, slows the release of mature rRNA from the nucleolus, and leads to the depletion of 18S and $28 S$ rRNAs. Together, these results suggest that the UPS is probably involved at many steps during ribosome biogenesis, including the maturation of the $90 \mathrm{~S}$ preribosome.
\end{abstract}

The nucleolus serves many functions $(4,23,36,51)$; however, its most prominent function remains ribosome biogenesis. This process comprises rRNA gene transcription; processing of the $47 \mathrm{~S}$ pre-rRNA to mature $18 \mathrm{~S}, 5.8 \mathrm{~S}$, and $28 \mathrm{~S}$ rRNAs; and assembly of preribosomal particles (21). Ribosome biogenesis is spatially organized in distinct compartments within the nucleolus. Transcriptionally active ribosomal genes are thought to be situated at either the boundaries of the fibrillar centers (FCs) and dense fibrillar components (DFCs) or in DFCs (45), whereas both the pre-rRNA processing and the assembly of preribosomal particles occur in the DFCs and granular components (GCs) (46). Ribosome biogenesis is regulated at multiple levels, including the transcription of ribosomal genes and the phosphorylation, methylation, and acetylation of component nucleolar factors, plus the trafficking and interaction of these factors (30).

\footnotetext{
* Corresponding author. Mailing address for James G. McNally: Laboratory of Receptor Biology and Gene Expression, 41 Library Dr., National Cancer Institute, Bethesda, MD 20892. Phone: (301) 4020209. Fax: (301) 496-4951. E-mail: monallyj@exchange.nih.gov. Mailing address for Nobuhiro Takahashi: Tokyo University of Agriculture and Technology, 3-5-8 Saiwai-cho, Fuchu-shi, Tokyo 1838509, Japan. Phone and fax: 81-042-367-5709. E-mail: ntakahas@cc .tuat.ac.jp.

$\dagger$ D.A.S. and M.K. contributed equally to this study.

$\$$ Supplemental material for this article may be found at http://mcb .asm.org/.

$\S$ Present address: Department of Cell Biology and Anatomy, Rosalin Franklin University of Medicine and Science, 3333 Green Bay Road, North Chicago, IL 60064.

I Present address: Department of Cell Biology, Institute of Experimental Medicine, Videnska 1083, Prague 4, Czech Republic.
}

Proteasomal regulation has been implicated in many processes, including cell cycle progression, transcription, and antigen processing $(24)(18,34,58)$. Indirect evidence has hinted at a possible role for the ubiquitin-proteasome system (UPS) in ribosomal biogenesis. Two different ubiquitination patterns have been reported for the late processing factor B23 $(25,48)$. Several other ribosomal factors may be ubiquitinated, as suggested by a recent proteomic analysis in yeast (37). It has also been known for many years that two ubiquitin precursors are ribosomal fusion proteins $(5,16,47)$. A large ribosomal subunit protein (L28) forms the most abundant ubiquitin-protein conjugate in yeast, and this modification is essential for ribosome function and efficient translation (53). A possible role for ubiquitin in nucleolus disassembly was also suggested (54). In addition, a ubiquitin ligase is known to regulate the processing and nuclear export of rRNA, as well as tRNA and mRNA in yeast (35). Finally, a temperature-sensitive point mutation of the $\mathrm{Cic} 1 \mathrm{p} / \mathrm{Nsa} 3 \mathrm{p}$ yeast protein, an adaptor for the $26 \mathrm{~S}$ proteasome, associates with early pre-60S particles (49), and inhibits both the synthesis of the mature $5.8 \mathrm{~S}$ and $25 \mathrm{~S}$ rRNAs and the release of pre-60S particles from the nucleolus (15). All of these data suggest a cross talk between the UPS and ribosome synthesis; however, little is known about how disruptions in UPS function affect ribosome biogenesis.

Here we demonstrate in mammalian cells that complexes associated with early and late pre-rRNA processing factors contain ubiquitinated components. Upon proteasome inhibition, we detected the accumulation of the $90 \mathrm{~S}$ preribosome, altered kinetics of some of the pre-rRNA processing factors, additional ubiquitination of complexes associated with late processing factors, changes in nucleolar morphology, and the 
inhibition of pre-rRNA processing. Our data suggest that the UPS is playing multiple roles in ribosome biogenesis, including 90S pre-ribosome maturation.

\section{MATERIALS AND METHODS}

Cell lines and plasmids. HeLa cells and a human kidney cell line 293EBNA (50) were used for most experiments. For the immunostaining experiments, two additional cell lines (MBA15 and 3617) were used. Cells were grown at $37^{\circ} \mathrm{C}$ with $5 \% \mathrm{CO}_{2}$ in Dulbecco modified Eagle medium (Gibco-BRL) supplemented with $2 \mathrm{mM}$ glutamine (Gibco-BRL) and $10 \%$ fetal bovine serum (HyClone). In preparation for the microscopy experiments, cells were transfected by electroporation (electroporator ECM 830; BTX) using $7 \mu \mathrm{g}$ of plasmid DNA and $15 \mu \mathrm{g}$ of sheared salmon sperm carrier DNA in a 2-mm gap cuvette at $200 \mathrm{~V}, 1-\mathrm{ms}$ pulse, four pulses, and 0.5-s intervals. After electroporation, the cells were plated in Lab-Tek II chambers (Nalgene), incubated overnight, and transferred to a phenol-red free medium to eliminate autofluorescence of the medium.

Green fluorescent protein (GFP)-UBF1, RPA194-GFP (12), fibrillarin-GFP, protein B23-GFP (13), GFP-GAR1 (41), GFP-U3-55k (40), GFP-Nopp140, GFP-NHPX (11), pEGFP-Rpp38 (26), NMD3-GFP (56), pEGFPhImp3, pEGFPhImp4, GFPhMpp10 (20), and hNop58-GFP (57) have been characterized previously. GFP-tagged rpS5 and rpL23 were kindly provided by S. Huang (Northwestern University Medical School, Chicago, IL). rpS27a-GFP was PCR amplified out of pcDNA (5) before it was subcloned into EGFP-N3 as an EcoRI-EcoRI fragment. FLAG-fibrillarin (59) and FLAG-nucleolin (60) have been described previously. DNA fragments of NNP-1 and B23 with a FLAG tag (DYKDDDDK) at their $\mathrm{N}$ terminus were amplified from a human placental cDNA library (ORIGENE, Technologies, Inc., Rockville, MD) by PCR using the primer sets 5'-ATTGAGCTAGCGCCACCATGGACTACAAGGACGACGA CGACAAGATGGT TTCGCGCGTGCAGCTC-3' and 5'-TTCTTCTTTGCG TCCCTCACTCCTAGGTGACT-3' for NNP-1 and 5'-ATATAGCTAGCGCC ACCATGGACTACAAGAACGACGACGACAAGGAAGATTCGATGGAC ATGGAC-3' and 5'-TATATGGATCCTTAAAGAGACTTCCTCCACTG-3' for B23, before subcloning into pcDNA3.1(+) vector (Invitrogen) as an NheIBamHI fragment. Hemagglutinin (HA)-ubiquitin was kindly provided by K. Nakayama (Kyusyu University, Medical Institute of Bioregulation, Fukuoka, Japan).

FRAP and FRAP analysis. For the fluorescence recovery after photobleaching (FRAP) experiments, cells were transfected with the GFP-tagged constructs, grown overnight in coverglass chambers (Lab-Tech), and transferred to a phenolred free medium before the experiment. For proteasome inhibition, either lactacystin $(50 \mu \mathrm{M})$ or MG-132 $(100 \mu \mathrm{M})$ (Calbiochem) was applied for $2 \mathrm{~h}$. In both cases, parallel control experiments with the vehicle $(0.1 \%$ [ $\mathrm{vol} / \mathrm{vol}]$ dimethyl sulfoxide [DMSO]) were conducted. FRAP and iFRAP experiments were carried out on a Zeiss 510 confocal microscope with a $100 \times 1.3$ N.A. oil immersion objective lens. Cells were kept at $37^{\circ} \mathrm{C}$ using an air-stream stage incubator (Nevtek). Since this system did not allow for a $5 \% \mathrm{CO}_{2}$ atmosphere, cells on the microscope stage were replaced every $\sim 30 \mathrm{~min}$ with a fresh chamber from the $\mathrm{CO}_{2}$ incubator. Bleaching was performed with the 488- and 514-nm lines from a $40-\mathrm{mW}$ argon laser operating at $75 \%$ laser power. Two iterations were used for the bleach pulse, which lasted $0.65 \mathrm{~s}$, and fluorescence recovery was monitored at low laser intensity $(0.2 \%$ of a $40-\mathrm{mW}$ laser $)$.

Data were analyzed using the statistical and graphical functions of SigmaPlot 7.0 (Sigma Chemical Co., St. Louis, MO). Approximately 10 separate FRAPs were performed and then averaged to generate a single FRAP curve. The standard error (SE) of the mean was used to statistically evaluate the difference between the control and treated groups.

RNA fluorescence in situ hybridization (FISH). Cells were transfected with the GFP-tagged B23 construct, grown overnight on 22-mm square coverslips each deposited on the bottom of a six-well plate, and treated with drug $(100 \mu \mathrm{M}$ MG-132 or $50 \mu \mathrm{M}$ lactacystin) for $2.5 \mathrm{~h}$ and fixed in $3.5 \%$ paraformaldehyde at room temperature for $15 \mathrm{~min}$. Cells were washed with phosphate-buffered saline (PBS) three times for $10 \mathrm{~min}$ each and then permeabilized for $10 \mathrm{~min}$ with $0.5 \%$ Triton X-100 in PBS.

For the hybridization, coverslips were air dried and placed on a glass slide containing a $20-\mu \mathrm{l}$ drop of hybridization mixture composed of $2 \times \mathrm{SSC}(1 \times \mathrm{SSC}$ is $0.15 \mathrm{M} \mathrm{NaCl}$ plus $0.015 \mathrm{M}$ sodium citrate), $25 \%$ formamide, $10 \%$ dextran sulfate, $1 \mathrm{mg}$ of tRNA/ml, and 5 to $10 \mu \mathrm{g}$ of probe DNA $/ \mathrm{ml}$. Rubber cement was used to seal the coverslips to the slide, and then the coverslips were left overnight at $37^{\circ} \mathrm{C}$.

Probe DNA was prepared with a biotin nick translation mix (Boehringer). The DNA templates were pA for the 5.8-28S pre rRNA (a 7-kb insert in pBR with EcoRI ends) and pB for the $18 \mathrm{~S}$ pre-rRNA (a 5.6-kb insert in pBR with EcoRI ends) (kindly provided by James E. Sylvester, Mayo Medical School, Rochester, $\mathrm{MN})$. The nick-translated DNA was denatured in $25 \%$ formamide at $95^{\circ} \mathrm{C}$ for 5 min and then placed on ice for 5 min before addition to the hybridization mixture. After hybridization, coverslips were washed for $15 \mathrm{~min}$ in $2 \times \mathrm{SSC}$ and $0.05 \%$ Triton X-100, washed again for $15 \mathrm{~min}$ in $2 \times \mathrm{SSC}$, and then washed for $5 \mathrm{~min}$ in $4 \times$ SSC. To detect hybridized probe, coverslips were incubated for $1 \mathrm{~h}$ with $2 \mu \mathrm{g}$ of avidin-rhodamine (Molecular Probes) $/ \mathrm{ml}$ in $4 \times \mathrm{SSC}, 0.1 \%$ bovine serum albumin, and $0.01 \%$ Tween 20 . The coverslips were then washed once for 10 min in $4 \times$ SSC with $0.05 \%$ Triton X-100 and then twice with just $4 \times$ SSC for 10 min each time, followed by a rinse in PBS, and then mounted in PBS on a slide. The DNA FISH slides were examined on a Leica DMRA Upright microscope with a Leica $100 \times 1.3$ N.A. oil immersion objective. Images were collected with a Photometrics Sensys charge-coupled device camera with binning of one to yield $0.067-\mu \mathrm{m}$ pixels.

Immunofluorescence. Cells were grown overnight on 22-mm-square coverslips and then treated with one of the proteasome inhibitors for $2 \mathrm{~h}$ and fixed with either absolute methanol at $-20^{\circ} \mathrm{C}$ for $15 \mathrm{~min}$ or in $3.5 \%$ paraformaldehyde at room temperature for $15 \mathrm{~min}$. After formaldehyde fixation, cells were permeabilized for 10 min with $0.5 \%$ Triton X-100 in PBS and washed in PBS three times for $10 \mathrm{~min}$ each time.

For all immunofluorescence experiments, cells were incubated overnight with the primary antibody diluted in PBS with $4 \%$ BSA and $0.1 \%$ Tween 20 . After incubation, cells were washed three times, for 10 min each time, in PBS and then incubated for 1 to $2 \mathrm{~h}$ with the appropriate secondary antibody, conjugated to either Texas Red or rhodamine. The cells were than washed three times more in PBS before final mounting in PBS and examination on a Leica DMRA microscope with a Leica $100 \times 1.3$ N.A. oil immersion objective. Images were acquired in green (GFP-GR) and red (antibody) fluorescence with a SenSys (Photometrics) camera with a KAF1400 chip configured to collect $0.067-\mu \mathrm{m}$ pixels. Experiments requiring a separation of the Texas Red and Cy5 antibody staining were carried out on a Zeiss META microscope with a Zeiss $100 \times 1.3$ N.A. oil immersion objective.

The following antibodies were used: antiubiquitin polyclonal antibody (AB1690; Chemicon International), antiubiquitin monoclonal antibody (RDIUBIQ-2C5; Research Diagnostics, Inc), anti-PML mouse monoclonal antibody (PG-M3, SC-960; Santa Cruz Biotechnology), and anticoilin mouse monoclonal antibody (C 1862; Sigma).

Ubiquitination assay, immunoprecipitation, and Western blot analysis. HAtagged ubiquitin $(20 \mu \mathrm{g} / 90-\mathrm{mm}$ dish) was cotransfected with FLAG-tagged expression plasmids $(20 \mu \mathrm{g} / 90-\mathrm{mm}$ dish) into 293EBNA cells by a calcium phosphate transfection method (22). Cultured cells (20 dishes) were collected in $1 \mathrm{ml}$ of hypotonic buffer (10 mM HEPES, $10 \mathrm{mM} \mathrm{KCl,} 10 \mathrm{mM} \mathrm{NaF}, 1 \mathrm{mM}$ phenylmethylsulfonyl fluoride) and lysed by adding IGEPAL CA-630 (Sigma Aldrich Chemical, Steinheim, Germany) to $0.1 \%$, followed by vortexing for $15 \mathrm{~s}$. Nuclei were collected by centrifugation at $1,400 \times g$ for 5 min, lysed in lysis buffer ( 50 $\mathrm{mM}$ Tris- $\mathrm{HCl}$ [pH 8.0], $150 \mathrm{mM} \mathrm{NaCl}, 0.5 \%$ IGEPAL CA-630, $1 \mathrm{mM}$ phenylmethanesulfonyl fluoride), and kept on ice for $30 \mathrm{~min}$. Nuclear extract was collected by centrifugation at $15,000 \mathrm{rpm}$ for $15 \mathrm{~min}$ at $4{ }^{\circ} \mathrm{C}$ and was analyzed on a 10 to $40 \%$ (wt/wt) sucrose gradient prepared in $50 \mathrm{mM}$ Tris- $\mathrm{HCl}-150 \mathrm{mM}$ $\mathrm{NaCl}-30 \mathrm{mM} \mathrm{MgCl}$ ( $\mathrm{pH}$ 7.5). Pre-ribosome particles were separated with a MLS-50 rotor (Beckman Coulter) at $45,000 \mathrm{rpm}(217,000 \times \mathrm{g})$ for $3 \mathrm{~h}$ at $4^{\circ} \mathrm{C}$. Pre-ribosomal fractions were detected at $254 \mathrm{~nm}$. Immunoisolation of pre-rRNP complexes associated with FLAG-tagged trans-acting factors and Western blot analysis were as described previously (60). Anti-HA antibody was from Sigma Aldrich Chemical. The primary antibodies used for Western blot analysis were goat anti-B23, goat antifibrillarin, and goat antinucleolin polyclonal antibodies (Santa Cruz Biotechnology; Santa Cruz, CA), anti-GAPDH monoclonal antibody (Ambion, Inc., Austin, TX), and anti-human NNP-1 antiserum (kindly provided by D. Hernandez-Verdun of Jacques Monod Institute, Paris, France). In some cases the staining intensity of the protein bands was quantified by a Kodak DS 1D Scientific imaging system (Kodak, New York, N.Y.).

Metabolic labeling and Northern blot analysis. For RNA analysis, HeLa cells were pretreated with $100 \mu \mathrm{M}$ MG-132 for $1 \mathrm{~h}$ and metabolically labeled for $3 \mathrm{~h}$ with $\left[{ }^{32} \mathrm{P}\right]$ orthophosphate (Perkin-Elmer) at a final concentration of $250 \mu \mathrm{Ci} / \mathrm{ml}$ in phosphate-free DMEM (Gibco). The ${ }^{32} \mathrm{P}$-labeled RNAs were isolated (RNAqueous Kit; Ambion, Inc.) and separated by electrophoresis on 1\% agarose formaldehyde gels. The RNAs were transferred to positively charged membranes (Ambion, Inc.). After UV cross-linking, autoradiography was performed with a PhosphorImager (Molecular Dynamics, Inc.).

BrUTP incorporation. HeLa cells transfected with GFP-tagged B23 as described above were grown overnight on 22-mm-square glass coverslips in a six-well plate and treated with either $100 \mu \mathrm{M}$ MG-132 or DMSO for $3 \mathrm{~h}$, and then the sites of active transcription were labeled in situ by BrUTP as previously 


\section{a}
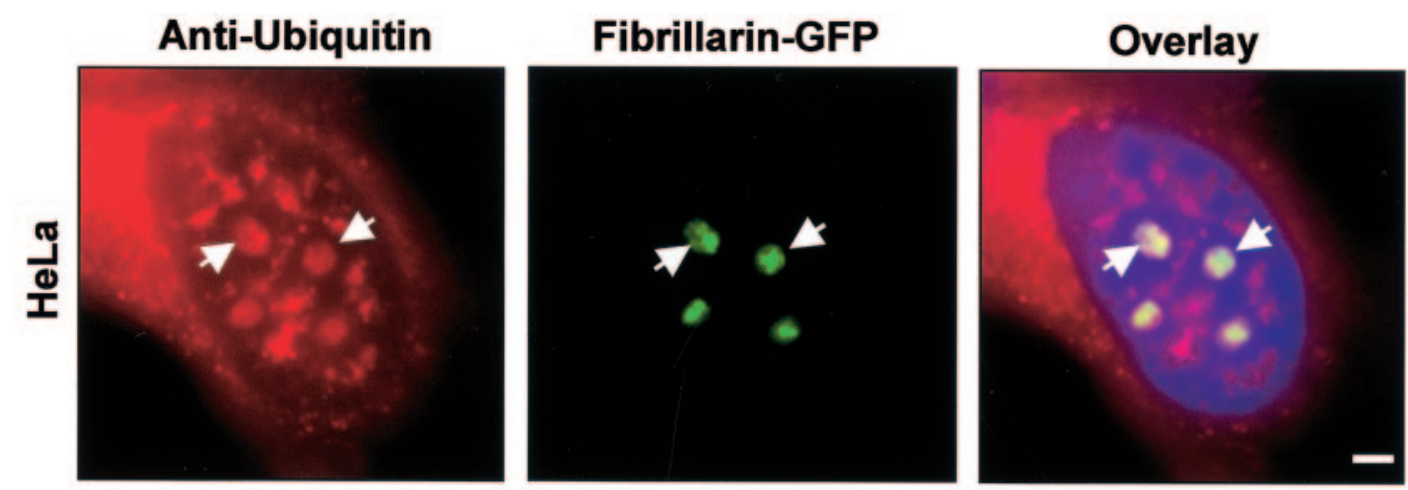

b
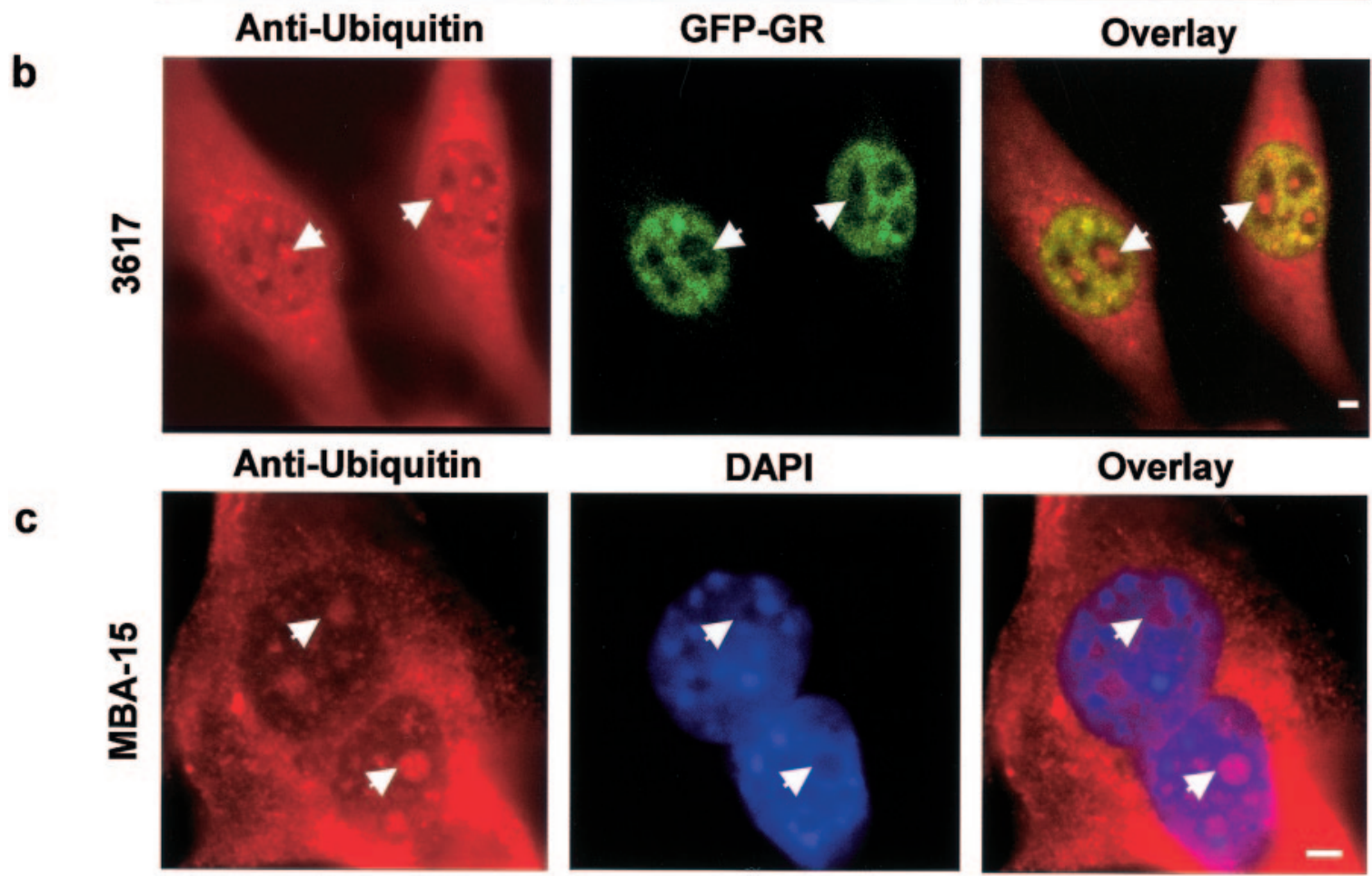

FIG. 1. Ubiquitin is present in the nucleolus, and the pre-rRNP complexes are ubiquitinated. (a) Immunofluorescence detection of ubiquitin in the nucleolus. Antibodies against ubiquitin stain the cytoplasm and nucleus as expected but also consistently colocalize with nucleoli, identified here in HeLa cells by fibrillarin expression. (b) 3617 cells, a derivative cell line from 3134, stably expressing GFP-tagged glucocorticoid receptor (GFP-GR) were also used (27). GFP-GR stains the nucleoplasm but is absent from nucleoli. These nucleoli are clearly stained with an anti-ubiquitin antibody. (c) In addition, MBA-15 cells also show a clear nuclear localization of ubiquitin as seen from the overlay with the DAPI DNA staining. Some of the nucleoli are indicated with arrows. Scale bar, $2 \mu \mathrm{m}$.

described (14). Briefly, cells were permeabilized in Tris-glycerol buffer $(20 \mathrm{mM}$ Tris-HCl [pH 7.4], $5 \mathrm{mM} \mathrm{MgCl}_{2}, 0.5 \mathrm{mM}$ EGTA, $25 \%$ glycerol, $5 \mu \mathrm{g}$ of digitonin/ $\mathrm{ml}, 0.5 \mathrm{mM}$ phenylmethylsulfonyl fluoride, and recombinant RNasin at $20 \mathrm{U} / \mathrm{ml}$ ) for $3 \mathrm{~min}$ at room temperature. The cells were then incubated for $5 \mathrm{~min}$ at $37^{\circ} \mathrm{C}$ in transcription buffer $(100 \mathrm{mM} \mathrm{KCl}, 50 \mathrm{mM}$ Tris- $\mathrm{HCl}[\mathrm{pH} 7.4], 5 \mathrm{mM} \mathrm{MgCl}, 0.5$ mM EGTA, 25\% glycerol, $2 \mathrm{mM}$ ATP, $0.5 \mathrm{mM}$ CTP, $0.5 \mathrm{mM}$ GTP, $0.5 \mathrm{mM}$ 5-bromouridine 5'-triphosphate from Sigma-Aldrich), $1 \mathrm{mM}$ phenylmethylsulfonyl fluoride, and recombinant RNasin at $20 \mathrm{U} / \mathrm{ml}$. The cells were fixed in $2 \%$ paraformaldehyde for $15 \mathrm{~min}$ and immunostained with an anti-bromouracil antibody (Caltag).

Microinjection of anti-28S rRNA oligonucleotide. An oligonucleotide complementary to 28S RNA in loop E9_1 (D-7a, C*GAGGGCAACGGAGGCCA $* \mathrm{C}$ GCCCG $* \mathrm{CCCT} * \mathrm{C}$ ) and labeled with fluorescein (43) was microinjected using a Microinjector System (Transjector 5246 \& InjectMan, Eppendorf, Germany) into HeLa cells pretreated with either $100 \mu \mathrm{M}$ MG-132 or the solvent (DMSO) for $2.5 \mathrm{~h}$ and incubated for $30 \mathrm{~min}$ at $37^{\circ} \mathrm{C}$. Fluorescently labeled nucleoli were subjected to inverse FRAP (iFRAP), wherein the whole nu- cleus except one of the nucleoli was bleached and the loss of fluorescence was monitored over time.

Electron microscopy. Cells were treated with MG-132 in DMSO $(100 \mu \mathrm{M})$ or with DMSO only (control cells) for 2 or $3.5 \mathrm{~h}$. Afterward, cells were fixed in $2.5 \%$ ( $\mathrm{vol} / \mathrm{vol}$ ) glutaraldehyde in $0.1 \mathrm{M}$ sodium cacodylate buffer $(\mathrm{pH} 7.4$ ) at $4^{\circ} \mathrm{C}$ for $1 \mathrm{~h}$, rinsed, postfixed in $1 \%(\mathrm{wt} / \mathrm{vol})$ osmium tetroxide in the same buffer for $30 \mathrm{~min}$, dehydrated, and embedded in Epon. After resin polymerization, ultrathin sections were stained with uranyl acetate $(30 \mathrm{~min})$, followed by lead citrate $(10 \mathrm{~min})$, and then examined with a Zeiss EM 900 electron microscope equipped with a KeenView camera using analySIS software (Soft Imaging System GmbH).

\section{RESULTS}

Ubiquitin is present within the nucleolus. Several proteins involved in ribosome biogenesis are ubiquitinated $(8,25,37$, 
a
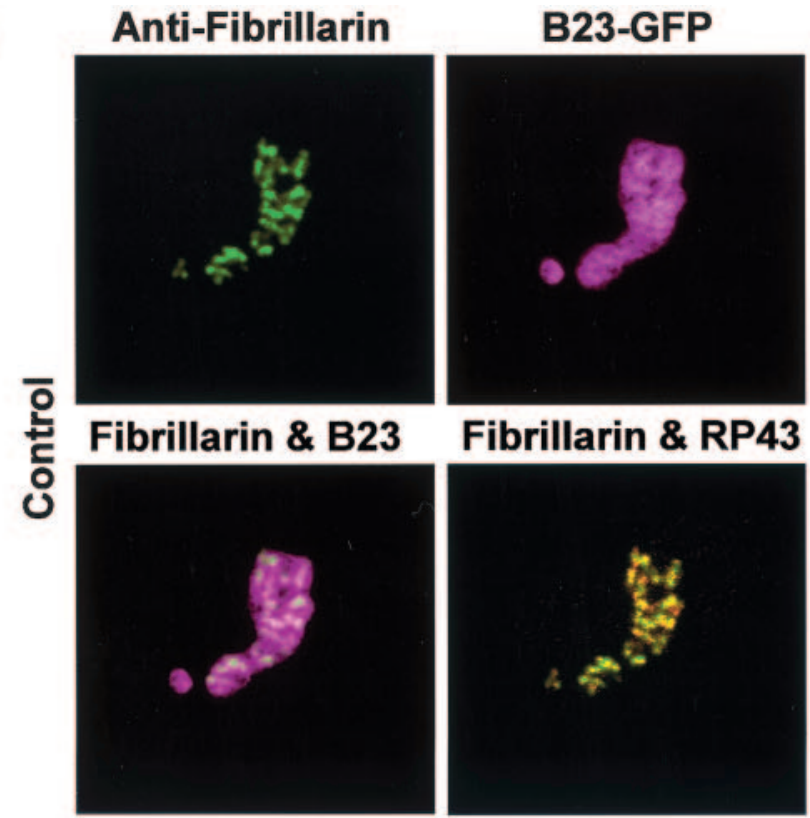

Fibrillarin \& RP43

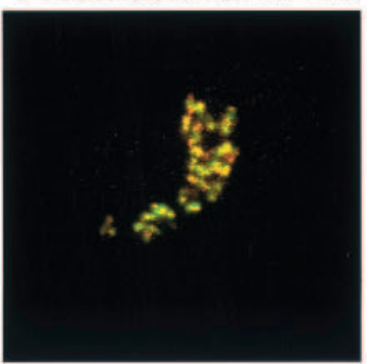

b
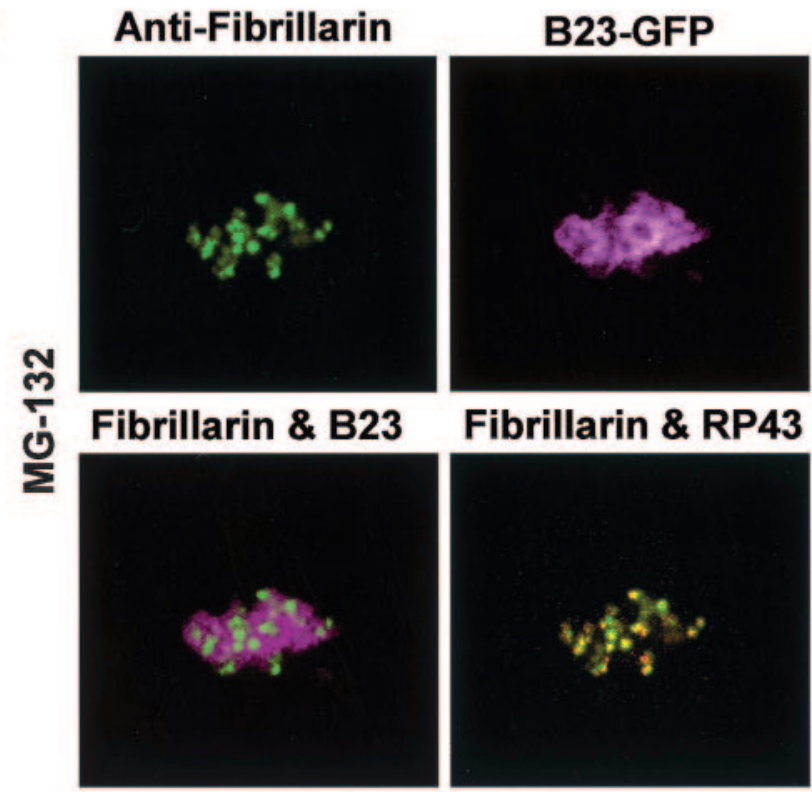

Fibrillarin \& RP43
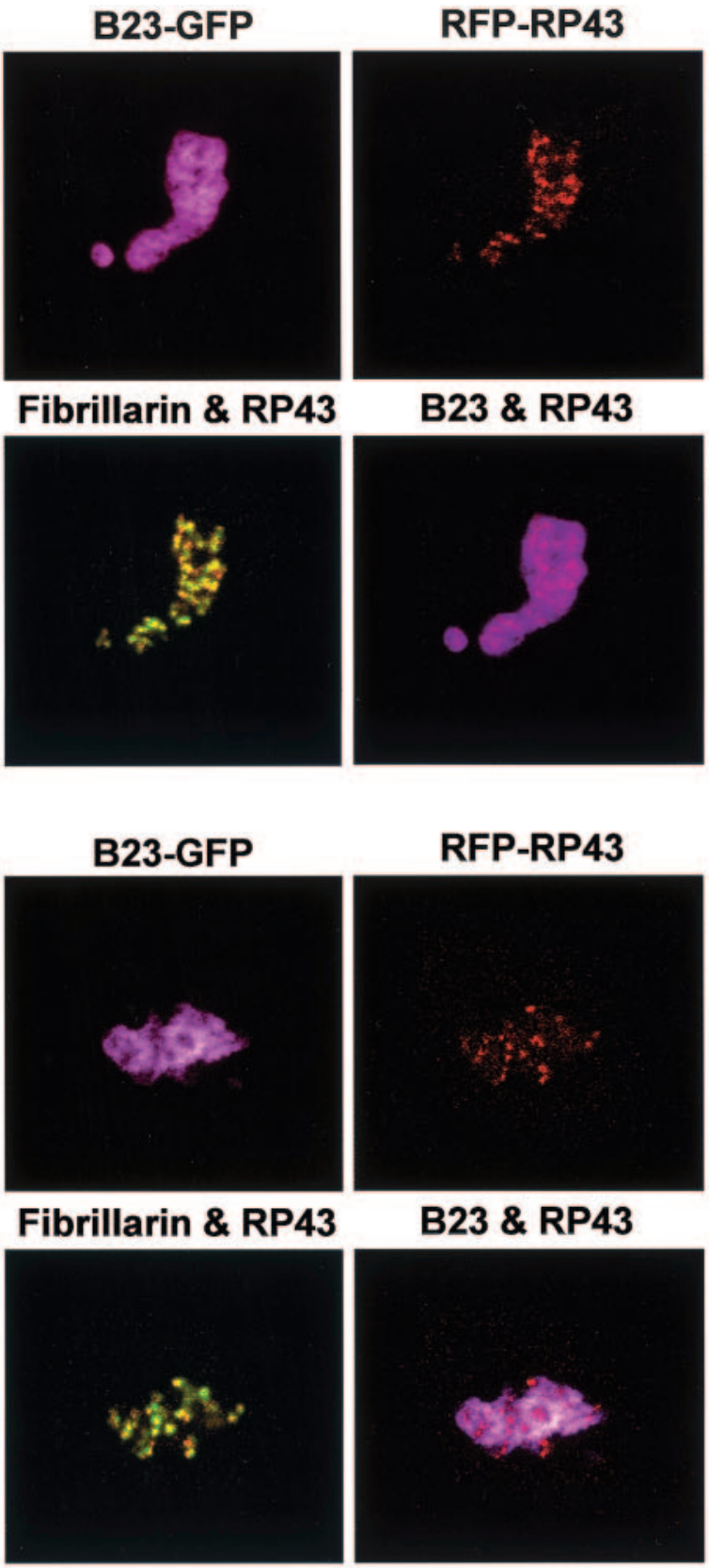

B23 \& RP43

C

Control

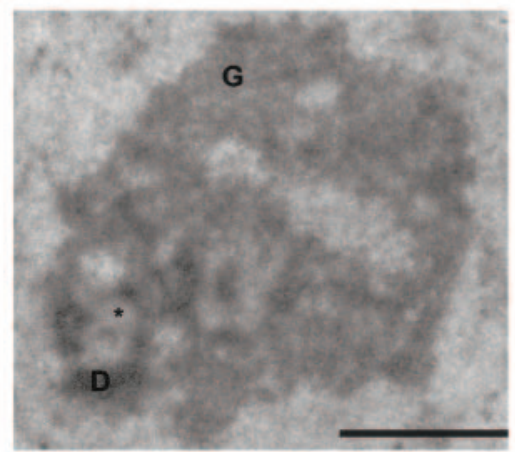

2 h MG-132

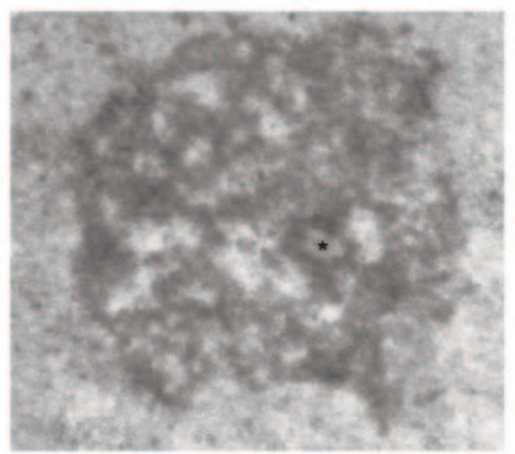

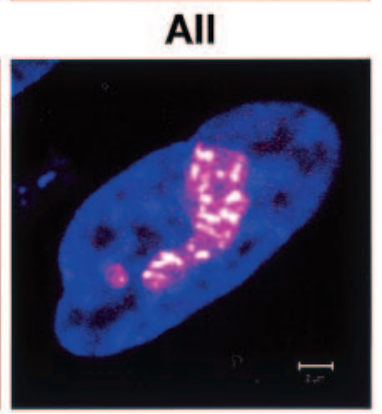

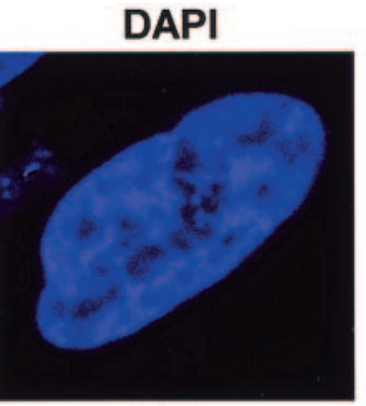

All

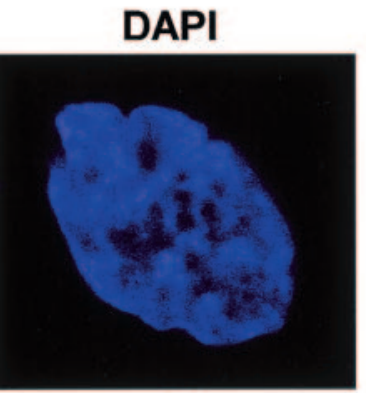

All

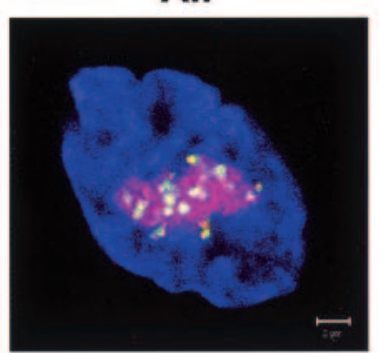

3.5 h MG-132 
48), but it is not known whether ubiquitinated proteins are present within the nucleolus. To test this directly, we used two different antiubiquitin antibodies to stain three different mammalian cell lines, and in each cell line we readily detected ubiquitin staining within nucleoli (Fig. 1). These observations demonstrate that ubiquitinated proteins are present in the nucleoli of mammalian cells.

Proteasome inhibition. To assess the role of the proteasome in nucleolar function and ribosome biogenesis, we used two specific proteasome inhibitors, MG-132 and lactacystin. Both were used because effects common to each are likely to reflect specific proteasomal functions (31). All of the experiments reported below were performed with MG-132, and critical results, as noted below, were repeated with lactacystin.

In pilot experiments, we tested different concentrations of these two inhibitors and different durations of treatment to identify conditions at which the first significant and reproducible effects of inhibition could be detected. In this way, we sought to identify the steps in ribosome biogenesis that are most dependent upon proteasome function. We found that these constraints were satisfied with a 2-h treatment with 100 $\mu \mathrm{M}$ MG-132 or $50 \mu \mathrm{M}$ lactacystin. Except as specifically noted, all data shown below are obtained from observations starting at $2 \mathrm{~h}$ after proteasome inhibition, and in some cases extended up to a maximum of $3 \mathrm{~h}$ when live cells were examined by light microscopy. Under these conditions, we also sought to determine whether two of the most dramatic effects reported below were reversible and found that they were following washout of the drug and reexamination of the cells $\sim 24$ h later (see Fig. S1 in the supplemental material).

Proteasome inhibition alters nucleolar morphology. As a first test of the effects of inhibition by MG-132 or lactacystin, we monitored changes in classic features of nucleolar morphology (Fig. 2), which are known to be a sensitive indicator of impaired nucleolar functions $(12,51)$. Three molecular markers were examined in HeLa cells: one for transcription (RP43), one for early preRNA processing (fibrillarin), and one for late preRNA processing (B23). As visualized alone, none of these three components exhibited dramatic changes in distribution after $2 \mathrm{~h}$ of proteasome inhibition (first rows in Fig. 2a versus $b$ ), except that the well-organized lattice-like structure of B23 in control cells appeared to partially disintegrate after proteasome inhibition.

However, changes were more clearly revealed by examining the relative locations of the different markers (second rows in Fig. 2a versus b). After proteasome inhibition, we found a distinct separation between the late processing factor B23 and the early processing factor fibrillarin (Fig. 2b), whereas in control cells these two factors exhibited considerable overlap (Fig. 2a). A similar relative redistribution was found for B23 and RP43 (Fig. 2a versus b). These results therefore suggest that proteasome inhibition induces specific and well-defined molecular changes in nucleolar structure.

Changes in nucleolar architecture were confirmed by electron microscopy. After 2 h of MG-132 treatment (corresponding to the duration of treatment in Fig. 2b), the nucleolar FCs and DFC were still visible, but the structure of the GC was extensively altered compared to control cells (Fig. 2c). After a longer treatment $(3.5 \mathrm{~h})$, the normal nucleolar structure was completely disrupted and DFCs and GCs could not be distinguished. Instead, a mass of undifferentiated electron-dense material was observed around structures resembling largerthan-normal FCs, suggesting that the effects observed after $2 \mathrm{~h}$ of proteasome inhibition were much more severe by $3.5 \mathrm{~h}$. As noted above, the experiments performed below were all done between 2 and $3 \mathrm{~h}$ after proteasome inhibition.

Differential effect of proteasome inhibition on nucleolar protein dynamics. The preceding results suggested that under our 2-h treatment conditions, proteasome inhibition could alter the relative distributions of selected nucleolar proteins. To examine this more systematically, we investigated the distributions of 16 proteins involved in different steps of ribosome biogenesis (rRNA gene transcription, early pre-rRNA processing, or late pre-rRNA processing). In addition to seeking to determine whether proteasome activity affects protein behavior in vivo, we also used FRAP to conduct a systematic survey of the mobility of those proteins. All but one of these analyses were performed with GFP fusion proteins whose functionality had been previously verified (see Materials and Methods).

We first analyzed the effect of proteasome inhibition on the distribution and mobility of two transcription factors UBF1 (upstream binding factor 1) and RPA194 (polymerase I catalytic subunit). After proteasome inhibition, both factors exhibited only small changes in their mobility and no detectable changes in their nucleolar distribution (Fig. 3a and b). These results are consistent with those above showing that proteasome inhibition had little effect on the distribution of another rRNA gene transcription factor RP43. We also found that proteasome inhibition had a modest effect on BrUTP incorporation within nucleoli (see Fig. S2 in the supplemental material). Together, these data suggest that under our conditions of proteasome inhibition, relatively small effects are observed on rRNA gene transcription.

Next, we examined nine early pre-rRNA processing factors and found three different classes of behavior in response to proteasome inhibition. The first class (NHPX, Mpp10, and U3-55K) was similar to the rRNA gene transcription factors and showed no change in mobility or localization after proteasome inhibition (Fig. 3c, d, and e). The second class (Nopp140 and GAR1) showed some reduction in mobility but no detectable changes in protein distribution (Fig. $3 f$ and g). The third class (fibrillarin, Imp4, Imp3, and Nop58) exhibited clearly

FIG. 2. Proteasome inhibition by MG-132 and lactacystin induces morphological changes in the nucleolus. Treatment of B23-GFP transfected HeLa cells with $100 \mu \mathrm{M}$ MG-132 or $50 \mu \mathrm{M}$ lactacystin (data not shown) for $2 \mathrm{~h}$ changes the relative distributions of B23 and fibrillarin, as well as B23 and RP43 (compare images in the second rows of panels a and b). Scale bar, $2 \mu \mathrm{m}$. The normal ultrastructural organization of the nucleolus is affected after treatment with $100 \mu \mathrm{M}$ MG-132 (c). Although structural counterparts of fibrillar centers (FC, asterisk) are observed both after $2 \mathrm{~h}$ and after $3.5 \mathrm{~h}$ of treatment, granular components $(\mathrm{G})$ and dense fibrillar components (D) gradually disintegrate. Scale bar, $1 \mu \mathrm{m}$. 

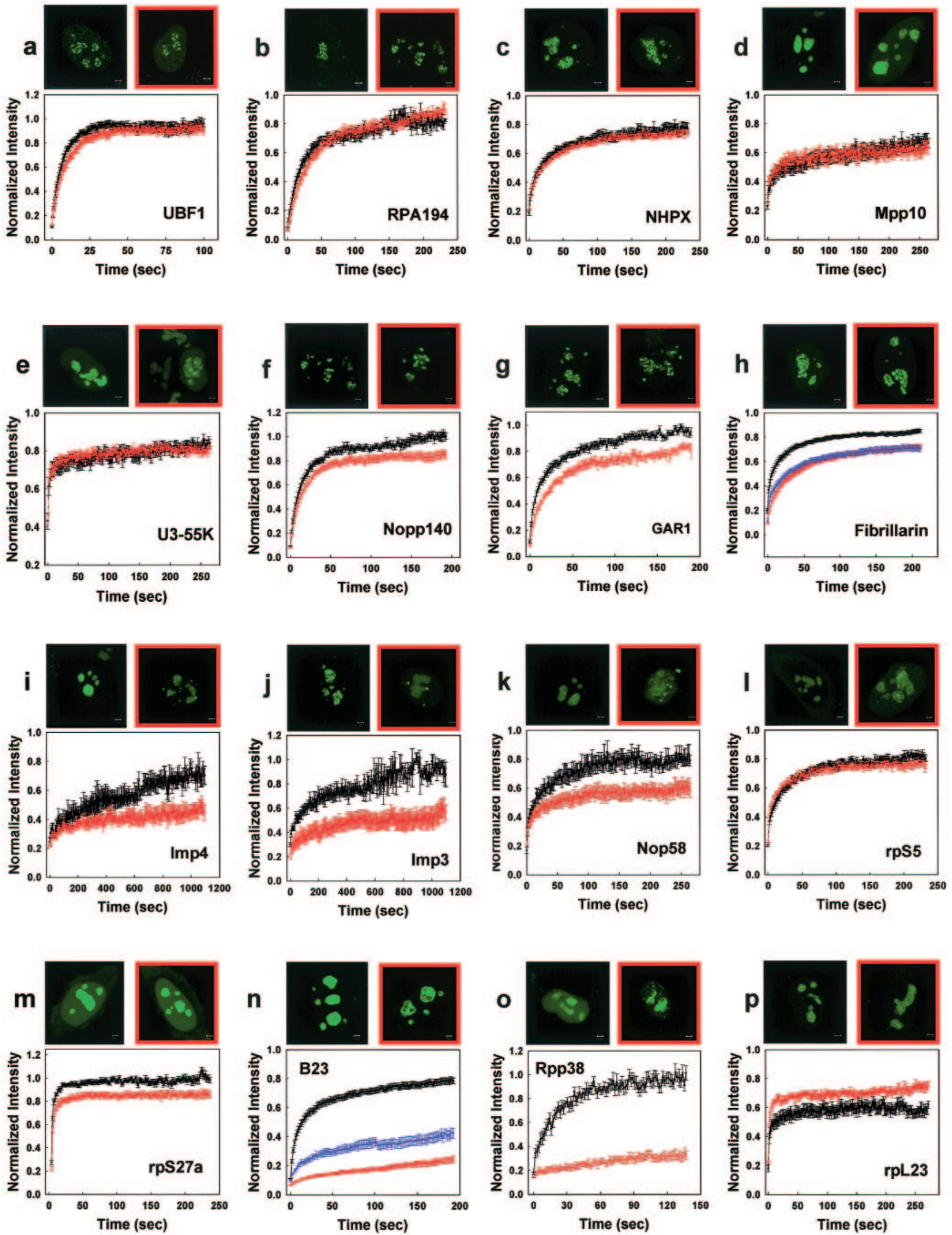
slower mobilities accompanied by distinctive changes in their distribution after proteasome inhibition (Fig. 3h, i, j, and k). (Note that under these conditions in which images were acquired between 2 and $3 \mathrm{~h}$ after proteasome inhibition, a more obvious defect in fibrillarin distribution could be detected compared to the 2-h treatment shown in Fig. 2.) Interestingly, three members of this latter class-Nop58, Imp3, and Imp4-accumulated into bright nuclear foci. For all three proteins, these foci were found to colocalize with Cajal bodies (CB), and for Imp4 and Imp3 some of the foci also colocalized with PML bodies (see Fig. S3 in the supplemental material). Imp3 foci showed a colocalization similar to that of Imp4 (data not shown).

Since many ribosomal proteins of the small subunit bind to the same complexes as the early processing factors (19), they are likely to exhibit a behavior similar to that of the early processing factors. Indeed, MG-132 treatment had no effect on the mobility of the small subunit protein $\mathrm{rpS} 5$ but reduced the mobility of another small subunit protein, rpS27a (Fig. 31 and $\mathrm{m})$.

We then investigated the effects of proteasome inhibition on two late processing factors B23 and Rpp38. The mobility of both factors was markedly disrupted, and the nucleolar distribution was also altered (Fig. 3n and o). Thus, in all cases where mobility changes were detected in either early or late processing factors, proteasome inhibition yielded reductions in mobility. This might reflect the aberrant formation of much larger complexes that diffuse more slowly or the failure of selected components to properly mature and exit particular nucleolar subcompartments at normal rates.

To complete our survey of the effects of proteasome inhibition on the distribution and mobility of nucleolar factors, we also examined a large ribosomal subunit protein rpL23 (Fig. $3 p$ ). Like the marked alterations in mobility of the late processing factors (B23 and Rpp38), the mobility of rpL23 was also significantly altered after proteasome inhibition but, interestingly, rpL23 mobility increased. This increased mobility could reflect reduced residence time at nucleolar sites that might arise due to aberrant production of the large subunit intermediates to which rpL23 normally binds. Consistent with such a possibility, we found that after proteasome inhibition the large subunit export factor NMD3 (56) no longer stained nucleoli, suggesting impaired production of the large ribosomal subunit (see Fig. S4 in the supplemental material).

Thus, we have shown that proteasome inhibition has selec- tive effects on the distribution and mobility of the factors involved in ribosome biogenesis.

Proteasome inhibition changes the structure of preribosome particles and induces accumulation of the $90 \mathrm{~S}$ preribosome. To directly assess the effect of proteasome inhibition on the level of $40 \mathrm{~S}, 60 \mathrm{~S}$, and $90 \mathrm{~S}$ preribosomes, we fractionated nuclear extract from control (DMSO) and MG-132-treated 293EBNA cells by sucrose gradient centrifugation. We found that a 2-h-long proteasome inhibition induced accumulation of the $90 \mathrm{~S}$ preribosome, while slightly decreasing the 40S and 60S levels (Fig. 4a and b). These data suggest that the UPS is specifically required for the further maturation of the $90 \mathrm{~S}$ pre-ribosome.

To assess under our treatment conditions whether the composition of the preribosome complexes was affected by proteasome inhibition, we compared the presence of early processing factors (fibrillarin and Nop52/NNP-1 [M. Kawasaki et al., unpublished data]) and late processing factors (B23 and nucleolin) in the preribosome fractions by Western blot analysis (Fig. $4 \mathrm{c}$ and $\mathrm{d}$ ). Consistent with the results of a mass spectrometry analysis of nucleoli following proteasome inhibition (1), we found a decrease in the overall level of these four processing factors in the combined $40 \mathrm{~S}, 60 \mathrm{~S}$, and $90 \mathrm{~S}$ preribosome fractions (Fig. 4e). Despite their overall decreased levels, two early processing factors (fibrillarin and NNP1) showed only a small change in their relative distribution among the 40S, 60S, and 90S preribosome fractions (Fig. 4f and g), while two late processing factors (nucleolin and B23) showed pronounced redistributions among these preribosome fractions (Fig. 4h to j). Specifically, we found that B23 levels decreased in the 90S and increased in the 40S fractions (Fig. 4 h), while nucleolin (which we found is present in two forms, a higher-molecular-weight form [NCL1] and a lower-molecular-weight form [NCL2]) levels decreased in $60 \mathrm{~S}$ and $90 \mathrm{~S}$ fractions and increased in $40 \mathrm{~S}$ fractions (Fig. 4c, d, i, and j). These results indicate that proteasome inhibition changes the preribosome particle composition and that the relative distribution of the late processing factors is more affected than the relative distribution of the early processing factors.

Proteasome inhibition differentially affects the ubiquitination of pre-rRNP complexes associated with early and late processing factors. Proteasome inhibition can lead to increased ubiquitination and/or stability of proteins that are normally targeted for degradation by the proteasome $(3,10,44)$. We therefore adopted this as an independent strategy to eval-

FIG. 3. Effects of proteasome inhibition on the kinetics of selected nucleolar factors. Proteasome inhibition has minimal effects on rRNA gene transcription factors. A treatment with $100 \mu \mathrm{M} \mathrm{MG}-132$ for up to $3 \mathrm{~h}$ does not affect the distribution or kinetics of UBF1 and RPA194, factors involved in Pol I transcription ( $\mathrm{a}$ and $\mathrm{b}$ ). Proteasome inhibition alters the mobility and cellular distribution of a subset of early pre-rRNA processing factors and small subunit ribosomal proteins. The kinetics of six (f to k) of nine (c to k) early processing factors were affected, whereas the remaining three did not show detectable changes. In addition, the cellular distribution of four of them (fibrillarin, Imp4, Imp3, and Nop58) was significantly altered (h to k). The mobility and distribution of one of the small subunit proteins (rpS5) was unaffected (1); however, the dynamics of another small subunit protein was reduced $(\mathrm{m})$. Proteasome inhibition radically alters the mobility of two late pre-rRNA processing factors and increases the mobility of a large subunit protein ( $\mathrm{n}$ to p). A 2- to 3-h treatment with either $100 \mu \mathrm{M} \mathrm{MG}-132$ or $50 \mu \mathrm{M}$ lactacystin leads to a dramatic change in the distribution of B23 and Rpp38 and drastic reduction in the mobility of both factors in nucleoli (n and o). In contrast the FRAPs of a large ribosomal subunit protein, rpL23, are faster than in control cells (p). Scale bars, $2 \mu \mathrm{m}$. The normal distribution of the proteins in HeLa cells is shown in black-framed pictures. The localization after proteasome inhibition is shown in red-framed pictures. Control FRAPs are shown in black, FRAPs after $100 \mu \mathrm{M}$ MG-132 treatment are shown in red, and FRAPs after the addition of $50 \mu \mathrm{M}$ lactacystin are shown in blue (error bars are \pm the SE). 
a

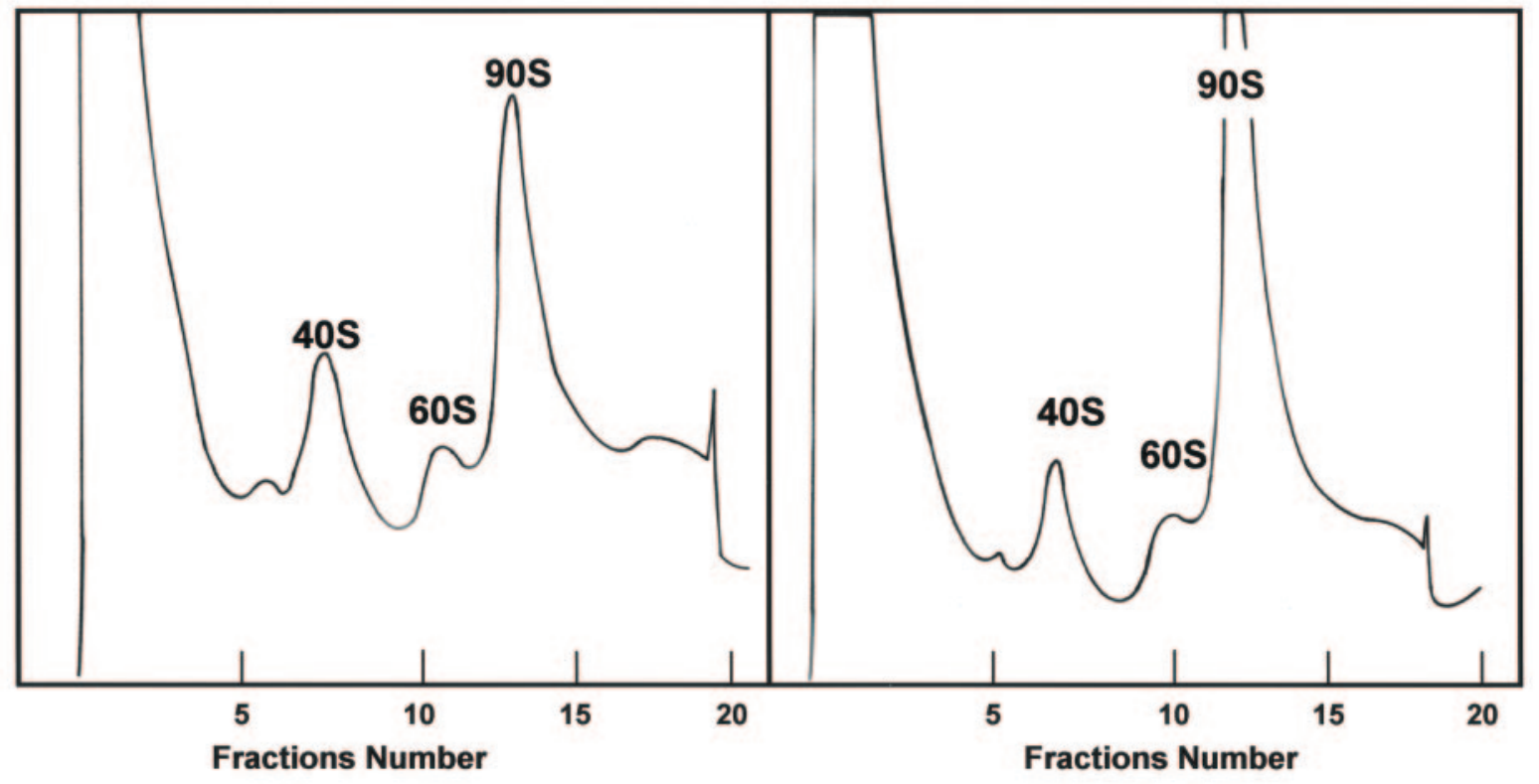

C

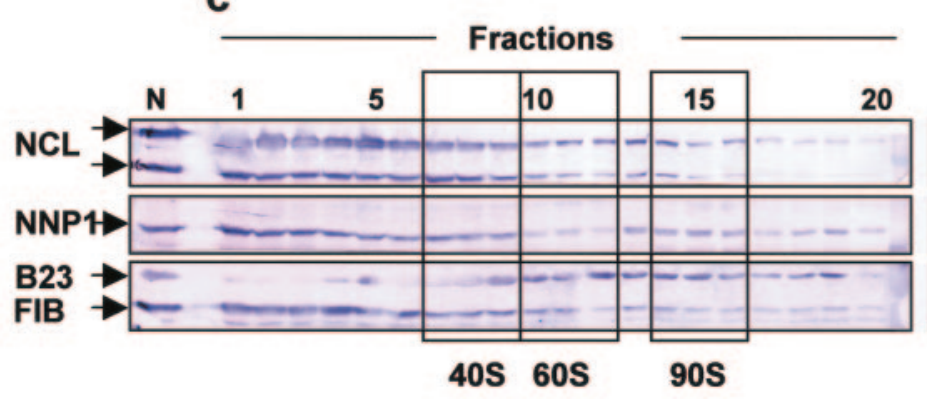

d

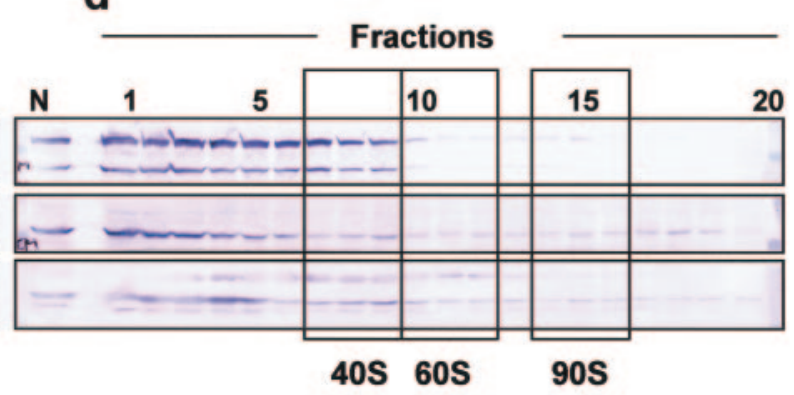

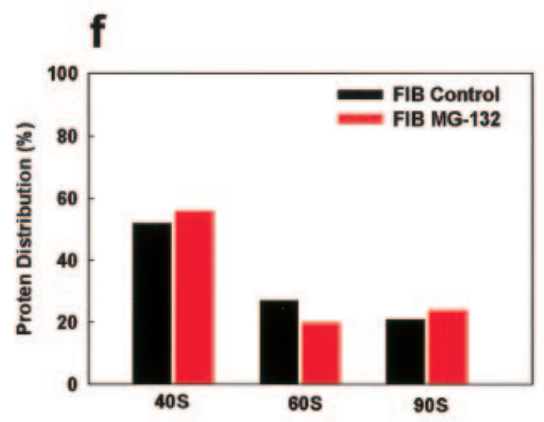
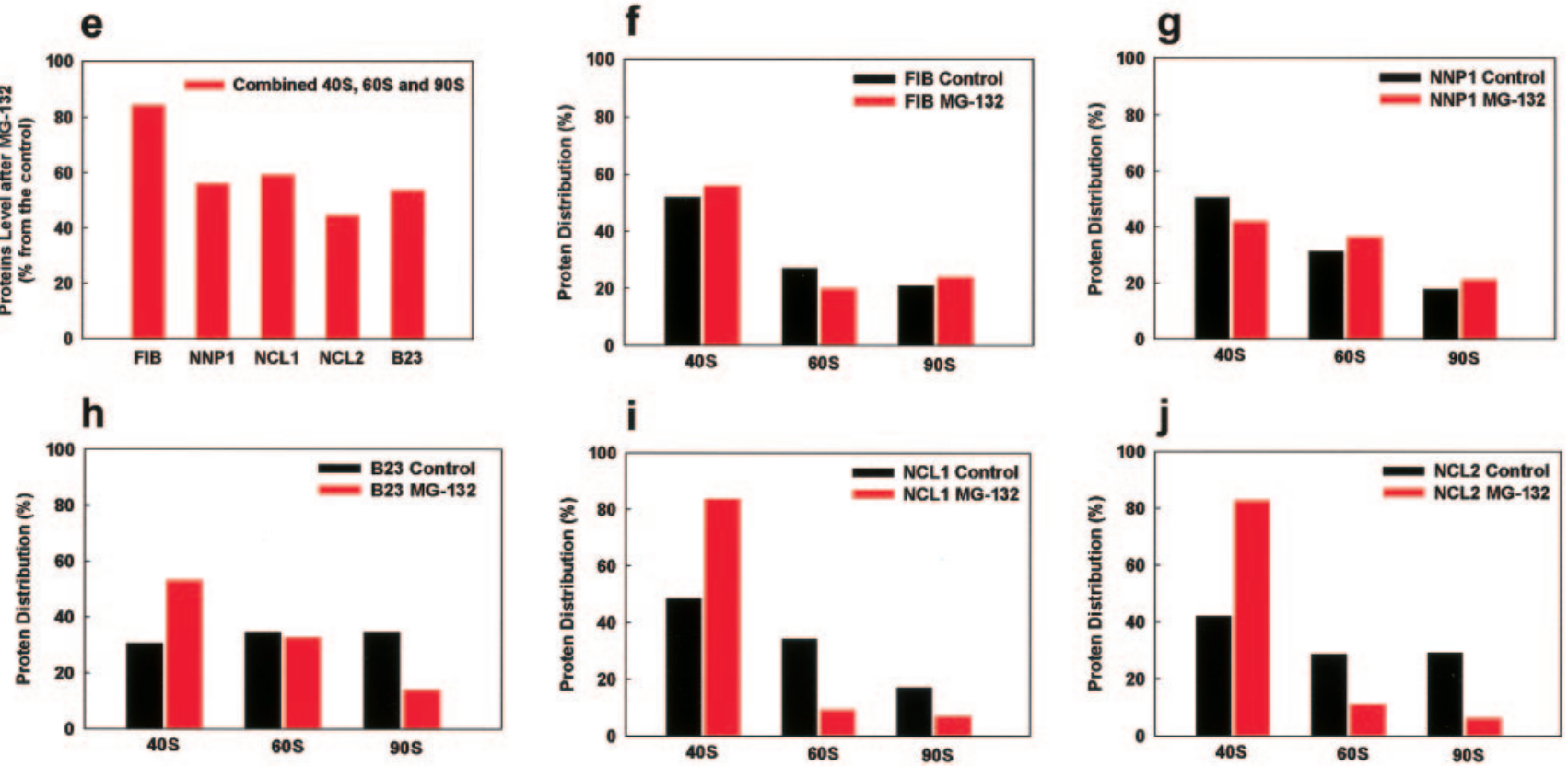

FIG. 4. Effects of proteasome inhibition on pre-rRNP complexes. Equal amounts of nuclear extracts from control (DMSO) or MG-132-treated 293EBNA cells were fractionated on a 10 to $40 \%$ sucrose gradient (a and b). MG-132 treatment (b) induces an accumulation of the 90S pre-ribosome $(143 \% \pm 13 \%)$ compared to the control (a). The $40 \mathrm{~S}$ and $60 \mathrm{~S}$ levels were less affected $(81 \% \pm 6 \%$ and $92 \% \pm 20 \%$, respectively). 
uate a possible role for proteasome function in ribosome biogenesis. Specifically, we examined ubiquitin levels before and after proteasome inhibition in selected pre-rRNP complexes associated with either early or late processing factors.

Complexes were isolated by using selected FLAG-tagged proteins: FLAG-fibrillarin and FLAG-Nop52/NNP-1 for complexes associated with early processing factors and FLAGnucleolin and FLAG-B23 for complexes associated with late processing factors. Cells were cotransfected with the FLAGtagged constructs and HA-tagged ubiquitin, and then complexes were immunoprecipitated using an anti-FLAG antibody. Components of the complexes were resolved by sodium dodecyl sulfate-polyacrylamide gel electrophoresis (SDSPAGE) and subjected to Western blot analysis with anti-HA and anti-FLAG antibodies, respectively (Fig. 5).

As a control to test whether this procedure yielded complexes that were derived from the preribosome fraction, nuclear extract from cells cotransfected with HA-tagged ubiquitin and one of the FLAG-tagged proteins (Nop52/NNP-1) was fractionated on a sucrose gradient to separate the 40S, 60S, and $90 \mathrm{~S}$ preribosome fractions (Fig. 6a and b). We found that each fraction contained FLAG-tagged Nop52/NNP-1, as assayed by a Western blot (Fig. 6d), suggesting that the Nop52/ NNP-1 complexes were in fact derived from the preribosome fraction. In addition, Western analysis with an anti-HA antibody indicated that ubiquitinated proteins were also present in these complexes (Fig. 6c), a finding consistent with our previous observations (Fig. 1) showing that even in the absence of proteasome inhibition, ubiquitinated proteins are found within the nucleolus.

With this evidence that our isolation procedure yielded complexes that could be found in the preribosome fractions, we then assayed the ubiquitination levels in these complexes before and after proteasome inhibition. Complexes associated with early processing factors (fibrillarin and Nop52/NNP-1) showed no change or even a slight decrease in ubiquitination after proteasome inhibition (higher-molecular-weight proteins in Fig. 5b). In contrast, complexes associated with late-processing factors (B23 and nucleolin) showed a slight increase in ubiquitination levels (Fig. 5b), raising the possibility that some components of these complexes may ordinarily be degraded by the proteasome. Similar results were obtained after lactacystin treatment (data not shown), suggesting that the effects are proteasome specific.

In addition, proteasome inhibition appeared to increase the amount of the pre-rRNP complex associated with B23 and perhaps also with nucleolin (compare the total protein levels in Fig. 5a for B23 and nucleolin with and without inhibition), suggesting a possible role for the UPS in their further maturation.

In sum, our results demonstrate that ubiquitin is present in pre-ribosomal complexes both before and after proteasome

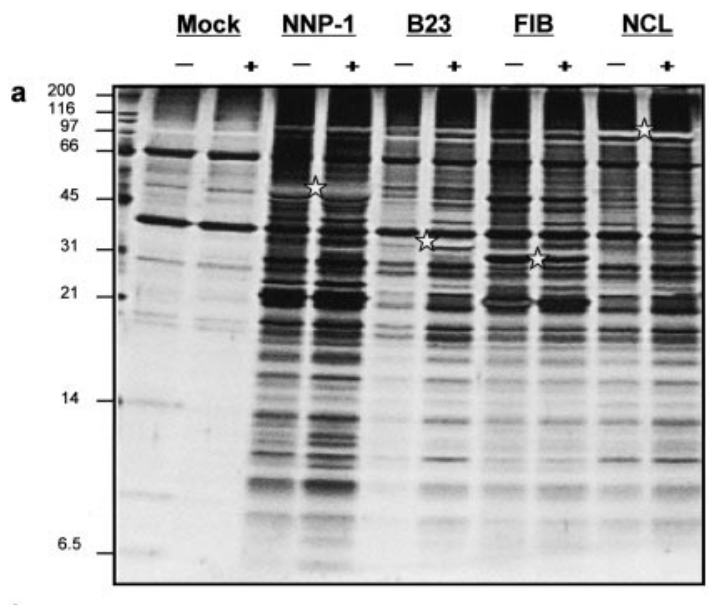

MG-132

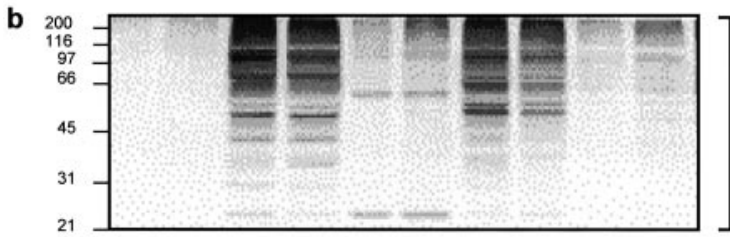

$[\mathrm{Ub}]_{\mathrm{n}}$

IB: anti-HA

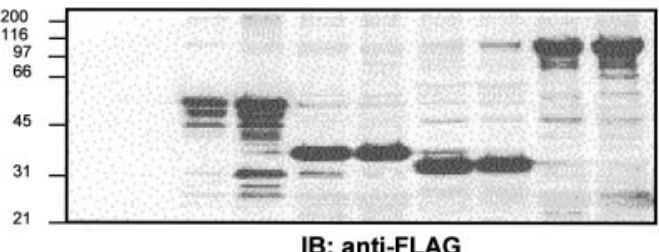

d

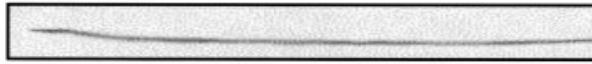

IB: anti-GAPDH

FIG. 5. Proteasome inhibition increases the ubiquitination of complexes associated with late processing factors. (a) Complexes isolated before (-) and after (+) MG-132 treatment. 293EBNA cells were cotransfected with HA-ubiquitin and FLAG-NNP-1, FLAG-B23, FLAGfibrillarin (FIB), or FLAG-nucleolin (NCL) as affinity baits. Complexes were separated by SDS-PAGE and silver stained. A star indicates the estimated location of the bait protein, based on its molecular mass as determined by the molecular mass standards (in kilodaltons) shown at the left side (see also panel c, which demonstrates the presence of the bait protein in each extract). (b and c) Western blots with anti-HA (ubiquitin tag) and anti-FLAG (bait tag) antibodies are shown. Mock, extract from cells transfected with the expression plasmid pcDNA3.1 without an inserted gene. (d) As an input control, the GAPDH content in each of the cell extracts is indicated at the bottom.

inhibition. Further, we found that this inhibition has a differential effect on the ubiquitin levels in complexes associated with early versus late processing factors. A slight increase was detected only with late processing factors (B23 and nucleolin). These observations are consistent with our FRAP analysis,

The data are the means of three independent experiments ( \pm the SE). Proteins in gradient fractions from control (a) and MG-132-treated 293EBNA cells (b) were further analyzed by immunoblotting with antinucleolin (NCL), anti-NNP-1, anti-B23, and antifibrillarin (FIB) antibodies (c and d). Three fractions from each peak representing the 40S, 60S, and 90S preribosomes were selected, and the staining intensity of each of the protein bands present in the areas surrounded by boxes was measured. Shown are changes in the proteins' level after proteasome inhibition relative to the control (e), as well as changes in the proteins' distribution among the $40 \mathrm{~S}, 60 \mathrm{~S}$, and $90 \mathrm{~S}$ preribosomal fractions (f to j). 


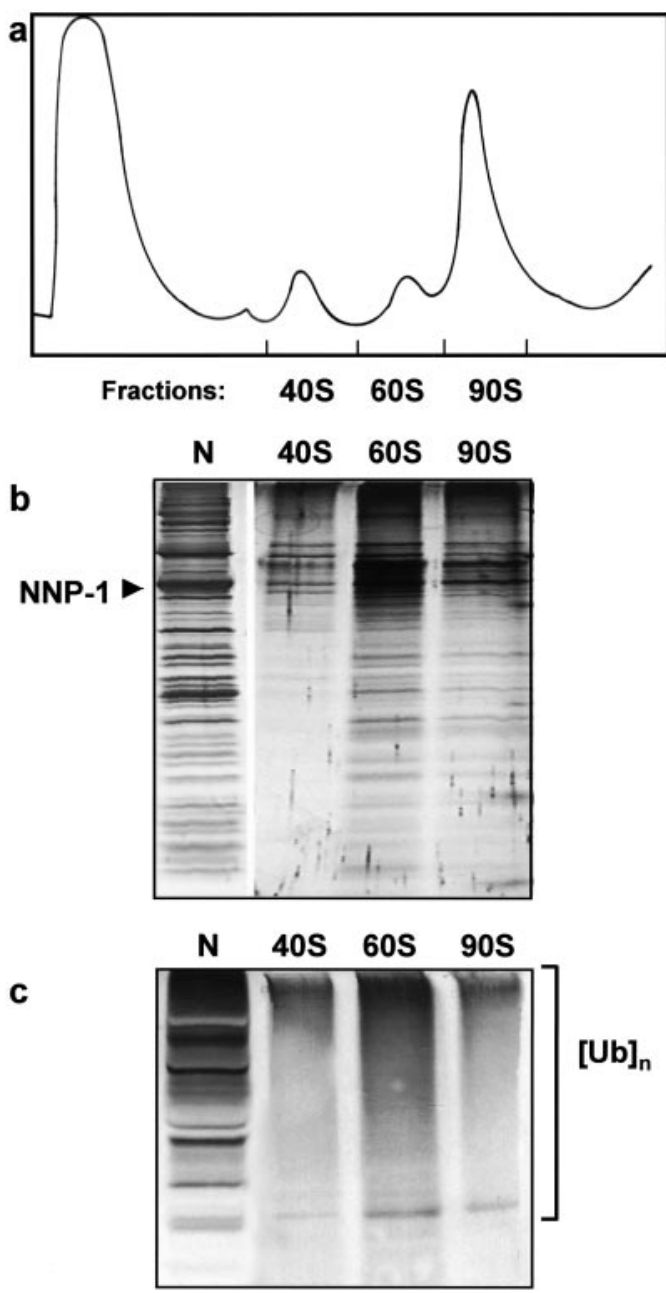

IB: anti-HA

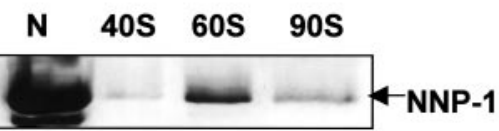

IB: anti-FLAG

FIG. 6. Immunoprecipitated complexes are parts of the pre-rRNP particles. (a) Nuclear extract prepared from the FLAG-NNP-1 and HA-ubiquitin cotransfected 293 EBNA cells was fractionated on a 10 to $40 \%$ sucrose gradient with continuous monitoring of the absorbance at $254 \mathrm{~nm}$. The FLAG-NNP-1-associated pre-rRNP complex was immunoisolated from each of the 40S, 60S, and 90S fractions; separated by SDS-PAGE (b); and analyzed by Western blotting with anti-HA (c) and anti-FLAG (d) antibodies, respectively. N, NNP-1-associated prerRNP complex isolated from FLAG-NNP-1-transfected cells.

where again the largest changes, in this case in protein mobility, were also detected with late processing factors (B23 and Rpp38). Together, these data provide two independent lines of evidence that proteasome inhibition exerts differential effects on the components involved in ribosome biogenesis.

Effects of proteasomal inhibition on the distribution of prerRNAs and on pre-rRNA processing. rRNAs are an integral part of the pre-rRNP complexes, and so we also investigated the effects of proteasome inhibition on these molecules. As a first step, we examined the effects of MG-132 (Fig. 7) on the distribution of $18 \mathrm{~S}$ and $28 \mathrm{~S}$ precursor RNAs.

After a 2-h proteasome inhibition, the distribution of both molecules changed in similar ways, although the extent of abnormality varied from cell to cell. These precursor RNAs tended to aggregate into clusters following proteasome inhibition. The severity of the clustering was consistently proportional to the degree of abnormality in the B23 distribution. In the most dramatic cases (in this example for 18S RNA, Fig. $7 \mathrm{a}$ ), clusters of the precursor RNA formed and were surrounded by a clearly spatially distinct B23 distribution. Less severe cases (in this example for 28S RNA, Fig. 7b), some evidence for clustering of the RNA was still apparent, and some spatial separation of B23 was also evident, but the differences between control cells were more subtle.

These disruptions in the distribution of the rRNAs could reflect underlying defects in the specific steps of pre-rRNA processing. To test this directly, cells were metabolically labeled with $\left[{ }^{32} \mathrm{P}\right]$ orthophosphate in the absence or presence of $100 \mu \mathrm{M}$ MG-132 (Fig. 8a). Consistent with the minimal effects detected on both transcription-factor distribution and mobility and also on BrUTP incorporation, we found that MG-132 had a small effect on the synthesis of native rRNA gene transcript (the levels of the native $47 \mathrm{~S}$ pre-rRNA were reduced by $15 \%$, even when the proteasome inhibition was carried out for up to $4 \mathrm{~h}$ (Fig. 8a and b). However, with 2- or 3-h proteasome inhibition treatments, ca. $50 \%$ less $36 / 32 \mathrm{~S}$ pre-rRNAs were produced. In addition, the $18 \mathrm{~S}$ and $28 \mathrm{~S}$ mature RNAs were nearly absent in MG-132-treated cells (Fig. 8a and b). These results demonstrate that proteasome inhibition significantly alters the pre-rRNA processing.

These data suggest that the formation of mature ribosomal subunits in the nucleolus is inhibited. To directly test that, we examined the effects of proteasome inhibition on rRNAs in vivo by injecting a fluorescently tagged oligonucleotide that specifically hybridizes with $28 \mathrm{~S}$ pre-RNA. This probe tags the mature 28S rRNA and all of its precursors (43).

To measure the rate at which the oligo-labeled transcript exits the nucleolus we used iFRAP. In this procedure, the entire nucleus except one nucleolus is bleached, and the rate of fluorescence loss from the unbleached nucleolus is measured. In untreated cells, nucleoli showed a steady loss of fluorescence, reflecting the production and export of mature $28 \mathrm{~S}$ rRNAs $\left(t_{1 / 2}=8.6 \mathrm{~s}\right)$ (Fig. 8c). In contrast, the MG-132-treated cells showed a much slower loss of fluorescence, a finding consistent with an accumulation of unprocessed pre-rRNA intermediates and impaired production and export of mature $28 \mathrm{~S}$ rRNA ( $t_{1 / 2}$ more than 9 min) (Fig. $8 \mathrm{c}$ ).

\section{DISCUSSION}

A number of previous studies have hinted at a cross talk between the UPS and ribosome production (15-17, 35, 47, 53). Lending further support to a functional link, we show here for the first time that ubiquitin is present within both the nucleolus and the pre-ribosomal complexes, identifying these as potential targets for the UPS-mediated regulation. By titrating the levels of inhibitors applied, we were able to disrupt selected processes while largely preserving others. Biochemical, structural, and in vivo mobility assays yielded a set of relatively consistent 

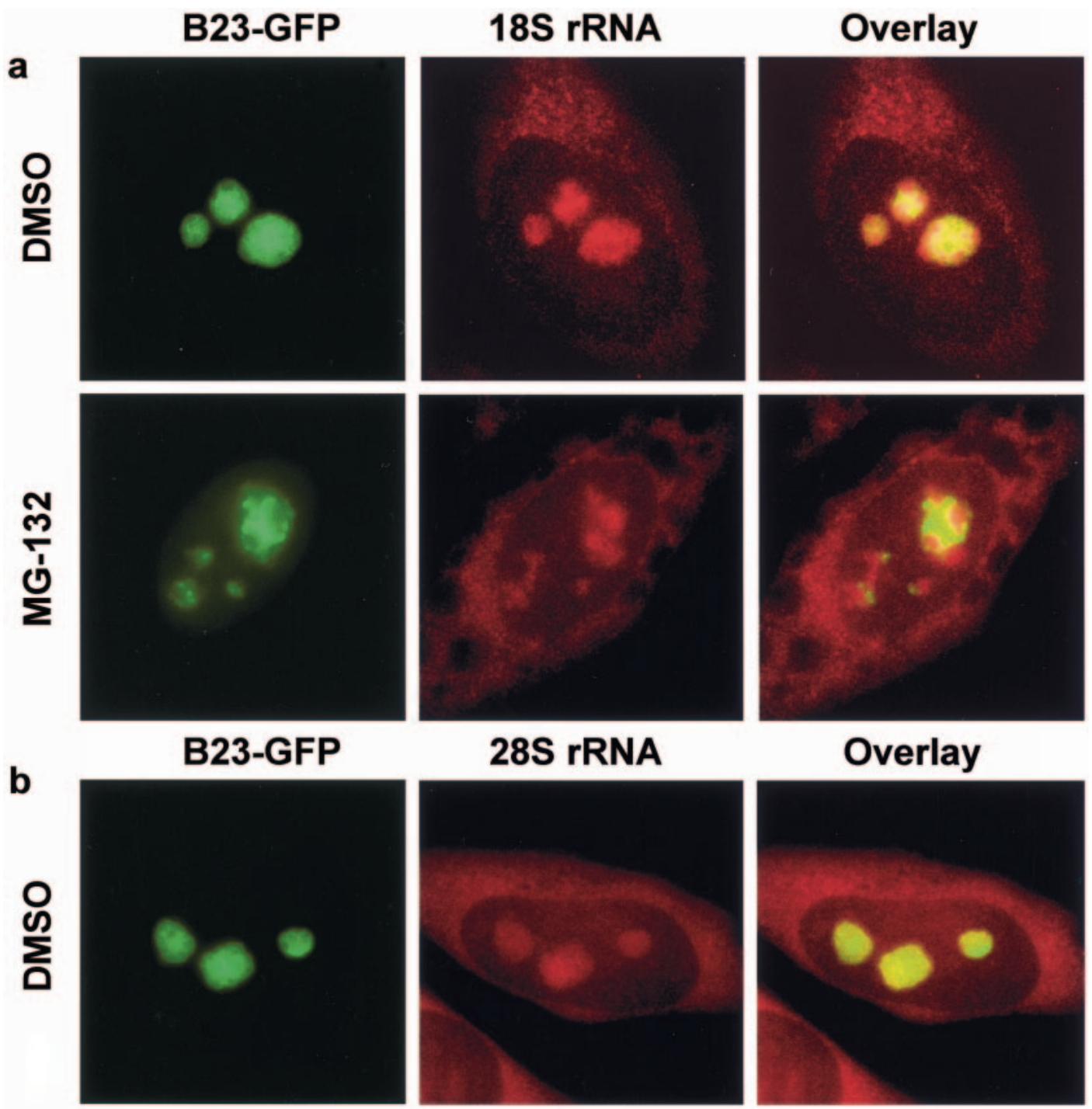

\section{S rRNA}
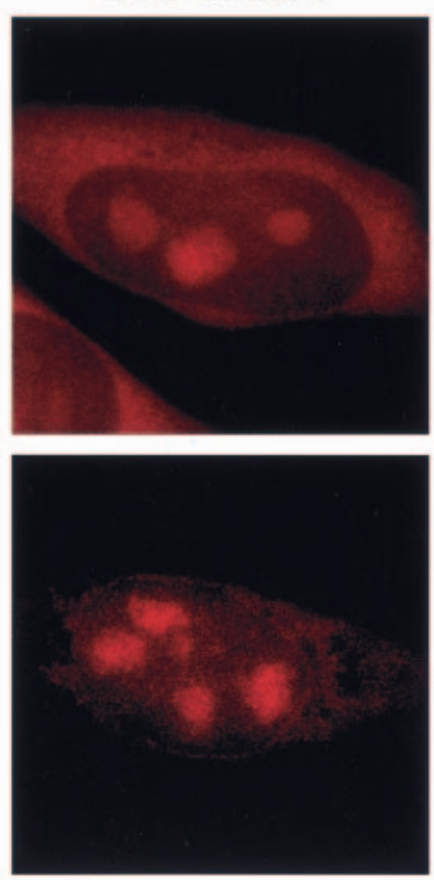

\section{Overlay}
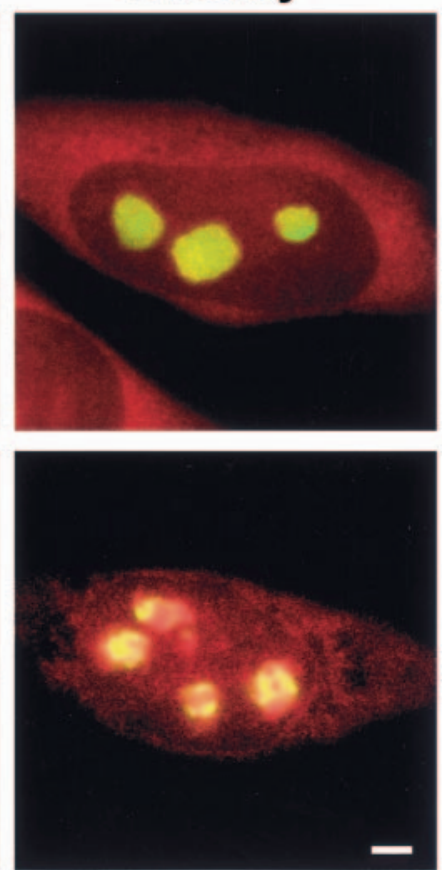

FIG. 7. Proteasomal inhibition alters the nucleolar distribution of both rRNA and rRNA precursors in HeLa cells. In the control (DHSO) both $18 \mathrm{~S}$ and 28S RNA FISH signals colocalize to a large extent with the B23 GFP-tagged construct (first rows in panels a and b). However, after proteasome inhibition (MG-132), both 18S rRNA and 28S rRNA signals exhibit a mixed pattern, with some areas colocalizing with B23 and others not (second rows in panels a and b). Note that the degree of mislocalization varied from cell to cell. The second row in panel a shows an example of a significant separation of the two signals, while the second row in panel b shows an example of a more modest effect. Scale bar, $5 \mu \mathrm{m}$.

results that implicated the most critical role for proteasomal function in the pre-rRNA processing and 90S pre-ribosome maturation. We discuss these observations in more detail below and their implications for a potential role of the UPS in ribosome biogenesis.
Nucleolar architecture is proteasome sensitive. We showed that the disruption of proteasome function has selective effects on some of the components of the nucleolar architecture. The distribution of factors associated with both rRNA gene transcription (UBF1 and RPA194) and certain early steps of rRNA processing 
a

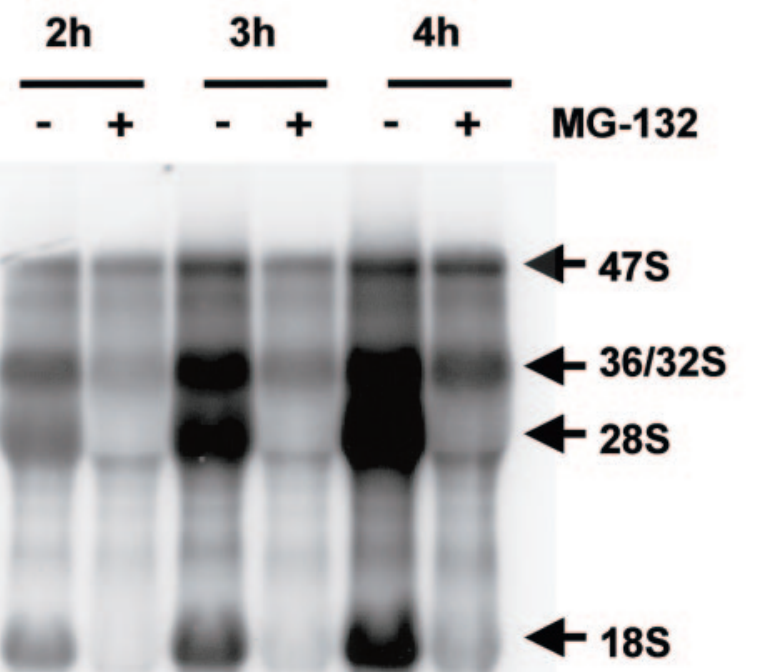

C
Control

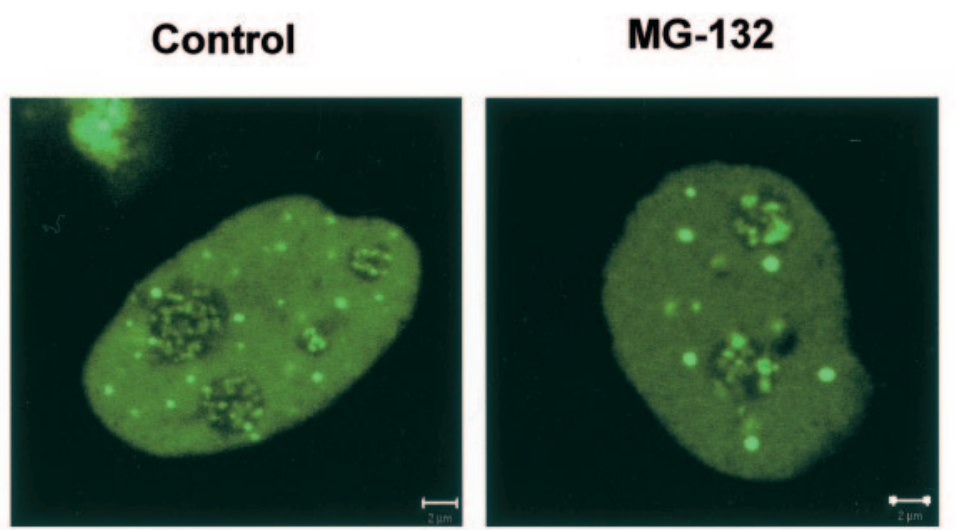

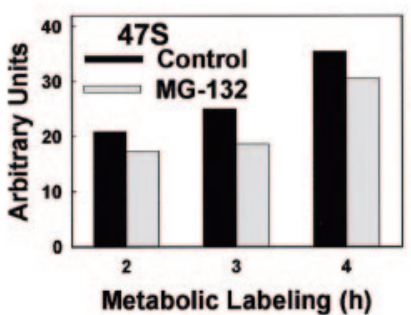
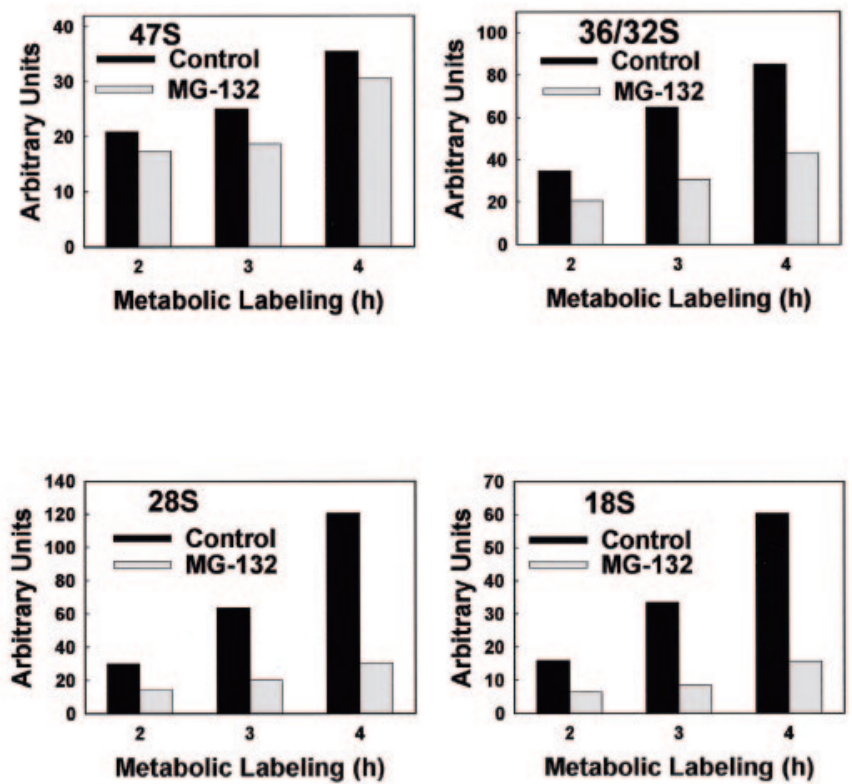

b

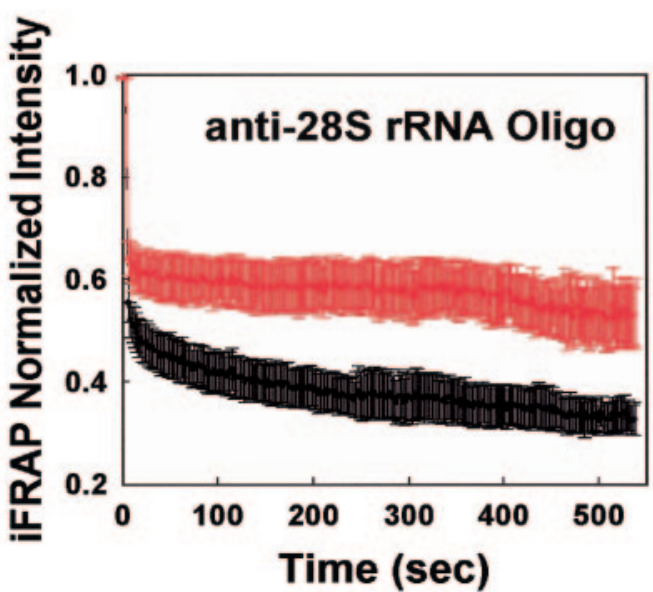

FIG. 8. Proteasomal inhibition affects pre-rRNA processing, and impairs the production of mature $28 \mathrm{~S}$. (a) ${ }^{32} \mathrm{P}$ metabolic labeling. Proteasome inhibition has modest effects on the levels of rRNA gene transcription (compare the levels of 47S in control and MG-132-treated cells), leads to reduced formation of $36 \mathrm{~S} / 32 \mathrm{~S}$ pre-rRNA, and impaired production of mature $28 \mathrm{~S}$ and $18 \mathrm{~S}$ rRNA. (b) Quantification of the ${ }^{32} \mathrm{P}-1 \mathrm{abeled} 47 \mathrm{~S}$ and $36 / 32 \mathrm{~S}$ pre-rRNAs and ${ }^{32} \mathrm{P}$-labeled mature $28 \mathrm{~S}$ and $18 \mathrm{~S}$ rRNAs corresponding to the representative experiment shown in panel a. (c) iFRAP experiments on an anti-28S rRNA oligonucleotide microinjected in control and MG-132-treated HeLa cells reveal a slower loss of 28S rRNA fluorescence from the nucleolus after proteasome inhibition. This indicates impaired production and export of mature 28S rRNA. Curves: black, control iFRAP; red, iFRAP after MG-132 treatment ( \pm the SE). Scale bars, $2 \mu \mathrm{m}$.

(U3-55K, NHPX, and Mpp10) was not altered. In contrast, we observed significant alterations due to proteasome inhibition in the distributions of selected early pre-rRNA processing factors (fibrillarin, Nop58, Imp3, and Imp4), late pre-
rRNA processing factors (Rpp38 and B23), and various rRNAs, including the $18 \mathrm{~S}$ and $28 \mathrm{~S}$ rRNAs, and their precursors.

Previous studies have shown that proteasome inhibition in- 
duces both accumulation of certain nuclear proteins in the nucleolus and accumulation of some nucleolar factors in the nucleoplasm $(1,2,6,8,32,42)$. Our results extend this work by demonstrating that selected components of nucleolar structure itself require the UPS to maintain their normal morphology. Consistent with the altered distributions of specific nucleolar proteins seen by light microscopy after proteasome inhibition, a comparable treatment yielded significant disruptions in nucleolar ultrastructure as detected by electron microscopy. After proteasome inhibition, fibrillar centers were still visible after $3.5 \mathrm{~h}$ of MG-132 treatment, but the other characteristic morphological components of the nucleolus, the dense fibrillar components, and especially the granular component were no longer distinguishable.

In sum, under our treatment conditions proteasome inhibition exerts specific effects on certain components of nucleolar architecture, and so we conclude that an intact UPS is required for normal nucleolar architecture.

Multiple steps of ribosome biogenesis are altered by proteasome inhibition. The altered nucleolar architecture described above is expected to reflect impaired ribosomal production, as the nucleolus is formed and maintained by the processes necessary to build ribosomes (33). Consistent with this, we found evidence that proteasome inhibition disrupts, at least to some degree, multiple processes in ribosome biogenesis, as we now summarize below.

(i) rRNA gene transcription. The process of ribosome production starts with rRNA gene transcription by polymerase I (Pol I). Based on the established role of the UPS in Pol II transcription (34), effects on Pol I transcription might also be expected. Consistent with this, Arabi et al. (2) detected a substantial effect on Pol I transcription, but this was based on a longer (4- or 8-h) MG-132 exposure. In contrast, Grandori et al. (19a), who used a 2-h treatment, found that Pol I transcription was not affected. In our experiments we found that a 2-h, 3-h, or even a 4-h treatment with MG-132 caused only a small, but reproducible decrease in rRNA gene transcription as assayed by metabolic labeling or by BrUTP incorporation. In addition, considering the blockage of pre-rRNA processing and the failure for pre-rRNA to accumulate after proteasome inhibition, it seems likely that Pol I transcription levels are at least slightly affected by proteasome inhibition.

(ii) Early and late processing. Of nine factors associated with early processing steps that we examined, three showed no detectable alteration in either their nucleolar distribution or in their in vivo mobility after proteasome inhibition, whereas six other early processing factors were disrupted in either their nucleolar distribution or their mobility (or both).

To provide more molecular and quantitative detail about the impact of proteasome inhibition on early processing steps, we measured the levels of the $90 \mathrm{~S}$ preribosome complex after proteasome inhibition and detected a significant increase. This increase is consistent with a mass spectrometry analysis of nucleoli (1), which showed that ribosomal proteins, particularly the small ribosomal subunit proteins, which are parts of the $90 \mathrm{~S}$ preribosome, accumulate in the nucleolus after proteasome inhibition. Our data now suggest that an intact UPS is required for further maturation and processing of the $90 \mathrm{~S}$ pre-ribosome.
Some of the late processing factors can also be found in the $90 \mathrm{~S}$ preribosome, even though they are also parts of the more mature complexes. In contrast to the early processing factors, whose relative distribution among the preribosome particles was largely preserved, the distribution of the late processing factors B23 and nucleolin (NCL) was significantly altered. In addition, the distribution of B23 and NCL in the 60S and especially the $40 \mathrm{~S}$ pre-ribosome fractions was also altered, suggesting that the UPS is involved in regulating the proper formation not only of $90 \mathrm{~S}$ but also of all of the preribosome subunits. We also observed striking alterations in the distribution and mobility of two late processing factors (B23 and Rpp38), as well as increases in the ubiquitination levels of complexes associated with two late processing factors (B23 and NCL). Together, these results suggest that the UPS likely influences steps in both early and late processing.

(iii) rRNA production. Our metabolic labeling data also show that proteasome inhibition induced significant defects in mature 28S rRNA production. Consistent with this, our RNA FISH experiments demonstrated an altered pre-rRNA distribution, and our iFRAP experiments suggested a failure to produce and export 28S rRNA after proteasome inhibition. These large effects could in principle arise from the accumulation of a series of smaller defects at earlier steps, or they might reflect a role for proteasomal activity on specific late processing factors-associated components.

Potential molecular mechanisms of the UPS in ribosome biogenesis. As detailed above, our results suggest that the UPS could be involved at multiple steps in ribosome biogenesis. As noted in the introduction, several proteins associated with ribosome biogenesis are ubiquitinated, and so these proteins are good candidates for molecules that might be regulated by the UPS.

An especially interesting candidate among these ubiquitinated proteins is B23. Previous studies have demonstrated that B23 polyubiquitination is associated with degradation of B23, inhibition of B23's endoribonuclease activity, and a concomitant impairment of ribosome biogenesis (25). Furthermore, B23 polyubiquitination is promoted by the tumor suppressor protein Arf, which is itself degraded by the proteasome (29). This degradation is likely to be important, since increased Arf levels are known to inhibit the processing of pre-rRNAs (55). Interestingly, in light of all of these data, we found that B23 showed some of the most profound alterations after proteasome inhibition, including significant disruptions in its mobility, distribution and relative levels in $40 \mathrm{~S}, 60 \mathrm{~S}$, and $90 \mathrm{~S}$ complexes. Together, these observations implicate B23 and Arf as two important molecules for which polyubiquitination and proteasome function are likely to be vital events in ribosome biogenesis.

However, our results after proteasome inhibition cannot be explained solely by an effect on B23 and Arf. Instead, it seems likely that there are other proteins involved in ribosome biogenesis yet to be identified that are also normally regulated by the UPS. In general, we can envision several such regulatory mechanisms.

Degradation by the UPS could normally prevent the incorporation of some polyubiquitinated and/or nonfunctional nucleolar factors into preribosome particles. In such a quality control scenario, inhibition of the proteasome would lead to 
incorporation of nonfunctional proteins into the pre-rRNP complexes and the prevention of subsequent pre-rRNA processing steps, as we observed here. Degradation in this case could occur within the nucleolus but, given the rapid shuttling of factors into and out of the nucleolus $(7,12)$, degradation could also occur anywhere in the nucleoplasm.

An alternate possibility is that proteasomal degradation is an obligatory step that is required as part of the normal process of pre-ribosomal complex maturation. In this scenario, the proteasome-mediated degradation would have to occur within the nucleolus. To date, it has not been possible to detect the $26 \mathrm{~S}$ proteasome within nucleoli $(38,58)$; however, proteasomemediated nucleolar degradation of survivin-deltaEx3 (52) has been reported, and we have also been able by immunostaining to detect the 19S regulatory particle in the nucleolus (see Fig. S5 in the supplemental material). Further studies will be required to determine unequivocally which specific nucleolar components are degraded and, if so, where the degradation occurs.

Still another possibility is that ubiquitination rather than degradation is the key step. In this scenario, ubiquitin moieties on key molecules could be playing a regulatory or signaling role akin to what has been found for some aspects of the $\mathrm{IKK} / \mathrm{NF}-\kappa \mathrm{B}$ pathway (28). Indeed, in addition to the evidence cited above implicating a role for $\mathrm{B} 23$ degradation in ribosome biogenesis, another recent study has demonstrated an alternate pathway for B23 polyubiquitination by BRCA1-BARD1 ubiquitin ligase (48). BRCA1-BARD1 polyubiquitination of B23 does not induce B23 degradation, an observation consistent with the possibility that some forms of polyubiquitination of B23 could serve some other signaling or regulatory function.

Biological relevance. Combined with the body of earlier evidence implicating connections between the UPS and ribosome biogenesis (see the introduction), our results now make it likely that the UPS is involved in multiple steps of ribosome biogenesis, including maturation of the $90 \mathrm{~S}$ pre-ribosome. These observations suggest some interesting biological consequences.

UPS regulation of ribosome biogenesis could provide a simple homeostatic mechanism whereby a reduction in protein degradation arising for any reason will lead to a drop in ribosome production and an accompanying reduction in protein synthesis. Such a reduction in protein degradation might occur under various natural conditions, but a particularly interesting and biologically relevant example occurs during cellular senescence. It is well established that proteasome function is impaired in aging cells $(9,39)$. It is also known that aging cells exhibit both structural changes in nucleoli and decreased ribosome production. Thus, our results now suggest that it is the impairment of proteasome function during senescence that could contribute to the deterioration in nucleoli and ribosome biogenesis detected in aging cells. This potential link could be tested more directly in the future by examining the effects of long-term, low-level proteasome inhibition on ribosome biogenesis and by applying some of the methods we used here to examine ribosome biogenesis in senescent cells.

\section{ACKNOWLEDGMENTS}

We thank S. Huang for GFP-tagged rpS5 and rpL23; S. Altman for pEGFP-Rpp38; G. Prujin for the GFP-tagged Imp3, Imp4, and Mpp10 plasmids; J. E. Sylvester for the rRNA gene-containing plasmids; J. C. Politz and T. Pederson for the anti-28S oligo; and I. G. Wool for the rpS27a cDNA. We also thank Robert Intine (NIH/NICHD) for helpful discussions during the preparation of the manuscript and Tatiana Karpova of the NCI Fluorescence Imaging Facility for assistance with the microscopy experiments.

The work of N. T. was supported by a Pioneer Research grant on Genome the Frontier from the Ministry of Education, Culture, Sports, Science, and Technology of Japan. The work of K.K. and I.R. was supported by grants of the Grant Agency of the Czech Republic (304/ 05/0374 and 304/04/0692), grant AVOZ50110509 from the Czech Academy of Sciences, and grants of the Ministry of Education, Youth, and Sports of the Czech Republic (MSM 0021620806 and LC535). This study was also supported by the Intramural Research Program of the NIH, National Cancer Institute, Center for Cancer Research.

\section{REFERENCES}

1. Andersen, J. S., Y. W. Lam, A. K. Leung, S. E. Ong, C. E. Lyon, A. I. Lamond, and M. Mann. 2005. Nucleolar proteome dynamics. Nature 433:77-83.

2. Arabi, A., C. Rustum, E. Hallberg, and A. P. Wright. 2003. Accumulation of c-Myc and proteasomes at the nucleoli of cells containing elevated c-Myc protein levels. J. Cell Sci. 116:1707-1717.

3. Boulanger, J., A. Vezina, S. Mongrain, F. Boudreau, N. Perreault, B. A. Auclair, J. Laine, C. Asselin, and N. Rivard. 2005. Cdk2-dependent phosphorylation of homeobox transcription factor CDX2 regulates its nuclear translocation and proteasome-mediated degradation in human intestinal epithelial cells. J. Biol. Chem. 280:18095-18107.

4. Carmo-Fonseca, M., L. Mendes-Soares, and I. Campos. 2000. To be or not to be in the nucleolus. Nat. Cell Biol. 2:E107-E112.

5. Chan, Y. L., K. Suzuki, and I. G. Wool. 1995. The carboxyl extensions of two rat ubiquitin fusion proteins are ribosomal proteins S27a and L40. Biochem. Biophys. Res. Commun. 215:682-690.

6. Chatterjee, T. K., and R. A. Fisher. 2003. Mild heat and proteotoxic stress promote unique subcellular trafficking and nucleolar accumulation of RGS6 and other RGS proteins: role of the RGS domain in stress-induced trafficking of RGS proteins. J. Biol. Chem. 278:30272-30282.

7. Chen, D., and S. Huang. 2001. Nucleolar components involved in ribosome biogenesis cycle between the nucleolus and nucleoplasm in interphase cells. J. Cell Biol. 153:169-176.

8. Chen, M., T. Rockel, G. Steinweger, P. Hemmerich, J. Risch, and A. von Mikecz. 2002. Subcellular recruitment of fibrillarin to nucleoplasmic proteasomes: implications for processing of a nucleolar autoantigen. Mol. Biol. Cell 13:3576-3587.

9. Chondrogianni, N., F. L. Stratford, I. P. Trougakos, B. Friguet, A. J. Rivett and E. S. Gonos. 2003. Central role of the proteasome in senescence and survival of human fibroblasts: induction of a senescence-like phenotype upon its inhibition and resistance to stress upon its activation. J. Biol. Chem. 278:28026-28037.

10. Dino, R. T., and A. von Mikecz. 2002. Proteasome-dependent processing of nuclear proteins is correlated with their subnuclear localization. J. Struct. Biol. 140:189-199.

11. Dundr, M., M. D. Hebert, T. S. Karpova, D. Stanek, H. Xu, K. B. Shpargel, U. T. Meier, K. M. Neugebauer, A. G. Matera, and T. Misteli. 2004. In vivo kinetics of Cajal body components. J. Cell Biol. 164:831-842.

12. Dundr, M., U. Hoffmann-Rohrer, Q. Hu, I. Grummt, L. I. Rothblum, R. D. Phair, and T. Misteli. 2002. A kinetic framework for a mammalian RNA polymerase in vivo. Science 298:1623-1626.

13. Dundr, M., T. Misteli, and M. O. Olson. 2000. The dynamics of postmitotic reassembly of the nucleolus. J. Cell Biol. 150:433-446.

14. Elbi, C., T. Misteli, and G. L. Hager. 2002. Recruitment of dioxin receptor to active transcription sites. Mol. Biol. Cell 13:2001-2015.

15. Fatica, A., M. Oeffinger, D. Tollervey, and I. Bozzoni. 2003. Cic1p/Nsa3p is required for synthesis and nuclear export of $60 \mathrm{~S}$ ribosomal subunits. RNA 9:1431-1436.

16. Finley, D., B. Bartel, and A. Varshavsky. 1989. The tails of ubiquitin precursors are ribosomal proteins whose fusion to ubiquitin facilitates ribosome biogenesis. Nature 338:394-401.

17. Glickman, M. H., and D. Raveh. 2005. Proteasome plasticity. FEBS Lett. 579:3214-3223.

18. Goldberg, A. L. 2003. Protein degradation and protection against misfolded or damaged proteins. Nature 426:895-899.

19. Grandi, P., V. Rybin, J. Bassler, E. Petfalski, D. Strauss, M. Marzioch, T. Schafer, B. Kuster, H. Tschochner, D. Tollervey, A. C. Gavin, and E. Hurt. 2002. 90 S pre-ribosomes include the $35 \mathrm{~S}$ pre-rRNA, the U3 snoRNP, and $40 \mathrm{~S}$ subunit processing factors but predominantly lack $60 \mathrm{~S}$ synthesis factors. Mol. Cell 10:105-115.

19a.Grandori, C., N. Gomez-Roman, Z. A. Felton-Edkins, C. Ngouenet, D. A. Galloway, R. N. Eisenman, and R. J. White. 2005. c-Myc binds to human ribosomal DNA and stimulates transcription of rRNA genes by RNA polymerase I. Nat. Cell Biol. 7:311-318. 
20. Granneman, S., J. E. Gallagher, J. Vogelzangs, W. Horstman, W. J. van Venrooij, S. J. Baserga, and G. J. Pruijn. 2003. The human Imp3 and Imp4 proteins form a ternary complex with hMpp10, which only interacts with the U3 snoRNA in 60-80S ribonucleoprotein complexes. Nucleic Acids Res. 31:1877-1887.

21. Hadjiolov, A. A. 1985 . The nucleolus and ribosome biogenesis, p. 268 Springer-Verlag, New York, N.Y.

22. Hayano, T., M. Yanagida, Y. Yamauchi, T. Shinkawa, T. Isobe, and N. Takahashi. 2003. Proteomic analysis of human Nop56p-associated pre-ribosomal ribonucleoprotein complexes: possible link between Nop56p and the nucleolar protein treacle responsible for Treacher Collins syndrome. J. Biol. Chem. 278:34309-34319.

23. Hernandez-Verdun, D., and P. Roussel. 2003. Regulators of nucleolar functions. Prog. Cell Cycle Res. 5:301-308.

24. Hershko, A., and A. Ciechanover. 1998. The ubiquitin system. Annu. Rev. Biochem. 67:425-479.

25. Itahana, K., K. P. Bhat, A. Jin, Y. Itahana, D. Hawke, R. Kobayashi, and Y. Zhang. 2003. Tumor suppressor ARF degrades B23, a nucleolar protein involved in ribosome biogenesis and cell proliferation. Mol. Cell 12:11511164.

26. Jarrous, N., J. S. Wolenski, D. Wesolowski, C. Lee, and S. Altman. 1999 Localization in the nucleolus and coiled bodies of protein subunits of the ribonucleoprotein ribonuclease P. J. Cell Biol. 146:559-572.

27. Kramer, P. R., G. Fragoso, W. Pennie, H. Htun, G. L. Hager, and R. R. Sinden. 1999. Transcriptional state of the mouse mammary tumor virus promoter can affect topological domain size in vivo. J. Biol. Chem. 274: 28590-28597.

28. Krappmann, D., and C. Scheidereit. 2005. A pervasive role of ubiquitin conjugation in activation and termination of IкB kinase pathways. EMBO Rep. 6:321-326.

29. Kuo, M. L., W. den Besten, D. Bertwistle, M. F. Roussel, and C. J. Sherr. 2004. N-terminal polyubiquitination and degradation of the Arf tumor suppressor. Genes Dev. 18:1862-1874.

30. Leary, D. J., and S. Huang. 2001. Regulation of ribosome biogenesis within the nucleolus. FEBS Lett. 509:145-150.

31. Lee, D. H., and A. L. Goldberg. 1998. Proteasome inhibitors: valuable new tools for cell biologists. Trends Cell Biol. 8:397-403.

32. Mattsson, K., K. Pokrovskaja, C. Kiss, G. Klein, and L. Szekely. 2001 Proteins associated with the promyelocytic leukemia gene product (PML)containing nuclear body move to the nucleolus upon inhibition of proteasome-dependent protein degradation. Proc. Natl. Acad. Sci. USA 98:10121017.

33. Melese, T., and Z. Xue. 1995. The nucleolus: an organelle formed by the act of building a ribosome. Curr. Opin. Cell Biol. 7:319-324.

34. Muratani, M., and W. P. Tansey. 2003. How the ubiquitin-proteasome system controls transcription. Nat. Rev. Mol. Cell. Biol. 4:192-201.

35. Neumann, S., E. Petfalski, B. Brugger, H. Grosshans, F. Wieland, D. Tollervey, and E. Hurt. 2003. Formation and nuclear export of tRNA, rRNA and mRNA is regulated by the ubiquitin ligase Rsp5p. EMBO Rep. 4:1156-1162.

36. Olson, M. O., K. Hingorani, and A. Szebeni. 2002. Conventional and nonconventional roles of the nucleolus. Int. Rev. Cytol. 219:199-266.

37. Peng, J., D. Schwartz, J. E. Elias, C. C. Thoreen, D. Cheng, G. Marsischky, J. Roelofs, D. Finley, and S. P. Gygi. 2003. A proteomics approach to understanding protein ubiquitination. Nat. Biotechnol. 21:921-926.

38. Peters, J. M., W. W. Franke, and J. A. Kleinschmidt. 1994. Distinct 19 S and $20 \mathrm{~S}$ subcomplexes of the $26 \mathrm{~S}$ proteasome and their distribution in the nucleus and the cytoplasm. J. Biol. Chem. 269:7709-7718.

39. Petropoulos, I., M. Conconi, X. Wang, B. Hoenel, F. Bregegere, Y. Milner, and B. Friguet. 2000. Increase of oxidatively modified protein is associated with a decrease of proteasome activity and content in aging epidermal cells. J. Gerontol. A Biol. Sci. Med. Sci. 55:B220-B227.

40. Pluk, H., J. Soffner, R. Luhrmann, and W. J. van Venrooij. 1998. cDNA cloning and characterization of the human U3 small nucleolar ribonucleo- protein complex-associated 55-kilodalton protein. Mol. Cell. Biol. 18:488498.

41. Pogacic, V., F. Dragon, and W. Filipowicz. 2000. Human H/ACA small nucleolar RNPs and telomerase share evolutionarily conserved proteins NHP2 and NOP10. Mol. Cell. Biol. 20:9028-9040.

42. Pokrovskaja, K., K. Mattsson, E. Kashuba, G. Klein, and L. Szekely. 2001. Proteasome inhibitor induces nucleolar translocation of Epstein-Barr virusencoded EBNA-5. J. Gen. Virol. 82:345-358.

43. Politz, J. C., R. A. Tuft, and T. Pederson. 2003. Diffusion-based transport of nascent ribosomes in the nucleus. Mol. Biol. Cell 14:4805-4812.

44. Qian, S. B., J. R. Bennink, and J. W. Yewdell. 2005. Quantitating defective ribosome products. Methods Mol. Biol. 301:271-281.

45. Raska, I. 2003. Oldies but goldies: searching for Christmas trees within the nucleolar architecture. Trends Cell Biol. 13:517-525.

46. Raska, I. 2004. Searching for active ribosomal genes. Prog. Mol. Subcell. Biol. 35:23-56.

47. Redman, K. L., and M. Rechsteiner. 1989. Identification of the long ubiquitin extension as ribosomal protein S27a. Nature 338:438-440.

48. Sato, K., R. Hayami, W. Wu, T. Nishikawa, H. Nishikawa, Y. Okuda, H. Ogata, M. Fukuda, and T. Ohta. 2004. Nucleophosmin/B23 is a candidate substrate for the BRCA1-BARD1 ubiquitin ligase. J. Biol. Chem. 279:30919-30922.

49. Saveanu, C., A. Namane, P. E. Gleizes, A. Lebreton, J. C. Rousselle, J. Noaillac-Depeyre, N. Gas, A. Jacquier, and M. Fromont-Racine. 2003. Sequential protein association with nascent $60 \mathrm{~S}$ ribosomal particles. Mol. Cell. Biol. 23:4449-4460.

50. Shen, E. S., G. M. Cooke, and R. A. Horlick. 1995. Improved expression cloning using reporter genes and Epstein-Barr virus ori-containing vectors. Gene 156:235-239.

51. Sirri, V., D. Hernandez-Verdun, and P. Roussel. 2002. Cyclin-dependent kinases govern formation and maintenance of the nucleolus. J. Cell Biol. 156:969-981.

52. Song, Z., and M. Wu. 2005. Identification of a novel nucleolar localization signal and a degradation signal in Survivin-deltaEx3: a potential link between nucleolus and protein degradation. Oncogene 24:2723-2734.

53. Spence, J., R. R. Gali, G. Dittmar, F. Sherman, M. Karin, and D. Finley. 2000. Cell cycle-regulated modification of the ribosome by a variant multiubiquitin chain. Cell 102:67-76.

54. Sudha, T., H. Tsuji, M. Sameshima, Y. Matsuda, S. Kaneda, Y. Nagai, F. Yamao, and T. Seno. 1995. Abnormal integrity of the nucleolus associated with cell cycle arrest owing to the temperature-sensitive ubiquitin-activating enzyme E1. Chromosome Res. 3:115-123.

55. Sugimoto, M., M. L. Kuo, M. F. Roussel, and C. J. Sherr. 2003. Nucleolar Arf tumor suppressor inhibits rRNA processing. Mol. Cell 11:415-424.

56. Trotta, C. R., E. Lund, L. Kahan, A. W. Johnson, and J. E. Dahlberg. 2003. Coordinated nuclear export of 60S ribosomal subunits and NMD3 in vertebrates. EMBO J. 22:2841-2851.

57. Verheggen, C., D. L. Lafontaine, D. Samarsky, J. Mouaikel, J. M. Blanchard, R. Bordonne, and E. Bertrand. 2002. Mammalian and yeast U3 snoRNPs are matured in specific and related nuclear compartments. EMBO J. 21:27362745 .

58. Wojcik, C., and G. N. DeMartino. 2003. Intracellular localization of proteasomes. Int. J. Biochem. Cell Biol. 35:579-589.

59. Yanagida, M., T. Hayano, Y. Yamauchi, T. Shinkawa, T. Natsume, T. Isobe, and N. Takahashi. 2004. Human fibrillarin forms a sub-complex with splicing factor 2-associated p32, protein arginine methyltransferases, and tubulins alpha 3 and beta 1 that is independent of its association with preribosomal ribonucleoprotein complexes. J. Biol. Chem. 279:1607-1614.

60. Yanagida, M., A. Shimamoto, K. Nishikawa, Y. Furuichi, T. Isobe, and N. Takahashi. 2001. Isolation and proteomic characterization of the major proteins of the nucleolin-binding ribonucleoprotein complexes. Proteomics 1:1390-1404. 